\title{
Diversity and delay performance of max link selection relay cooperation systems over non-identical Nakagami- $m$ fading channels
}

\author{
Xiangdong Jia ${ }^{1,2,3^{*}}$, Meng Zhou ${ }^{1}$, Xiaochao Dang ${ }^{1}$, Longxiang Yang ${ }^{2,3}$ and Hongbo Zhu ${ }^{2,3}$
}

\begin{abstract}
The work focuses on the max link selection (MLS) schemes of buffer-aided relaying over the independent and non-identically distributed (i.ni.d) Nakagami- $m$ fading channels in terms of outage probability, average packet delay, and improved diversity-delay trade-off schemes. By modeling the transition of buffer states as a Markov chain, we first obtain the outage probability and average packet delays for MLS schemes, which are given with closed-form expressions. The achieved results show that the i.ni.d fading channels impose severe loss in the diversity and coding gains of MLS schemes. Especially, when the disparity among the channel powers is very large, the diversity order provided by the MLS schemes is less than the ones provided by the traditional best relay selection (T-BRS) and max-max link selection (MMLS) schemes even if the buffers' size is large enough. For the average packet delay of MLS schemes, our results show that they are impacted by the transmit power and the fading severity factors. In general, in the low transmit power, the average packet delays are varying with the transmit power. However, in the high transmit power, the average packet delays approach the stationary values. Besides of this, it is also achieved that the i.ni.d fading makes the average packet delays of MLS schemes at all relays are different. When the powers of relay-destination links are greater than the ones of source-relay links, the average packet delays can be reduced evidently. Motivated by the observations, a weight-based MLS (W-MLS) diversity-delay trade-off scheme is proposed. The proposed W-MLS diversity-delay trade-off schemes not only have the low packet delays at all relays but also provide enough diversity and coding gains over T-BRS and MMML, which can overcome the performance loss caused by non-identical distribution on MLS schemes.
\end{abstract}

Keywords: Cooperative relay, Buffers, Non-identical fading, Nakagami-m, Outage performance, Packet delay

\section{Introduction}

Recently, cooperative diversity has received much attention from academia and industry due to its ability to increase the throughput, coverage, and reliability of wireless networks $[1,2]$. It is based on the broadcast nature of wireless medium. In cooperative diversity systems, each node can act as a relay and helps the other

\footnotetext{
* Correspondence: jiaxd@nwnu.edu.cn

${ }^{1}$ College of Computer Science and Engineering, Northwest Normal University, Lanzhou 730070, China

${ }^{2}$ Wireless Communication Key Lab of Jiangsu Province, Nanjing University of Posts and Telecommunications, Nanjing 210003, China

Full list of author information is available at the end of the article
}

nodes of the networks to forward their information to their respective destination nodes so that the spatial diversity is exploited. The relay cooperation channels were first investigated by van der Meulen [3]. While the works [4-8] investigated the three-node relay channel, the work [9] considered the multi-relay cooperation ones, where the best relay selection scheme has been proposed (that also referred as traditional best relay selection (T-BRS)). All these works have proved that cooperative systems offer outstanding throughput gains over non-cooperative ones [10-13].

However, due to the practical half-duplex (HD) constraint, in traditional protocols, the relays employ a

\section{Springer}


prefixed schedule for transmission and reception, which is independent from the quality of the transmitting and receiving channels in a round of communications. The prefixed transmission and reception schedule limits the exploitation of achievable diversity degrees. Moreover, for multi-relay cooperation schemes, only the same relay node is selected for receiving data from source and forwarding data to destination in a round of communications. This prefixed schedule and the selection of the same relay in a round of communications may lead significant performance degradation in wireless systems where the qualities of the transmitting and receiving channels significantly vary with time, since it may prevent the relays from exploiting the best transmitting and the best receiving channels. Clearly, the performance of the HD relaying systems could be improved if the above constrains are relaxed. For example, by relaxing the prefixed schedule constraint for data reception and transmission, the link with the highest quality could be always used in each time slot. Similarly, by relaxing the constraint of the same relay employed in a round of communications, the systems can always select the relays with the strongest source-relay $(S-R)$ channel and the strongest relay-destination $(R-D)$ channel for reception and transmission, respectively. To achieve the ends, it is only possible if the relays are equipped with a buffer and can store data packets before retransmitting them later at a suitable time slot.

Early works of buffer-aided relaying systems can be founded in [14] and [15], where a three-node relaying system was considered and a data buffer was introduced at relay node. Due to the prefixed transmission and reception schedule abandoned, the results in [14] and [15] showed that the utilization of data buffer at relay can improve the throughout of relaying systems. The literature [16] focused on the multi-antenna relaying system with buffer at relay and pointed out that if the numbers of antennas at source and destination are equal or larger than the number of antennas at relay, the buffer-aided half-duplex systems can outperform the ideal full-duplex ones. Motivated by the works [14] and [15], the buffer-aided relay systems have been investigated widely such as in [17-25]. Later, in [26] and [27] the idea of buffer-aided relaying was expanded to multiple relay systems, where the authors have proposed the max-max link selection (MMLS) protocol firstly. Specially, to exploit the quality of relay channels as possible, the MMLS schemes abandon the restriction of using the same relay for reception and transmission in a round of communications. Because the strongest links from both $S-R$ and $R-D$ group channels are always selected for data transmission, respectively, the MMLS scheme has significant coding gain over the traditional max-min scheme [12]. However, the results in [26] and [27] show also that the diversity gain achieved by the max-max selection schemes is still the same as the one achieved by the T-BRS schemes owing to a prefixed transmission and reception schedule employed, such that the available diversity degree being not full exploited. Therefore, to overcome this prefixed schedule constraint and to improve the diversity gain of buffer-aided relay selection systems, in [28], the maxlink selection (MLS) scheme has been proposed. In the MLS schemes, the constraint on the prefixed transmission and reception order is further relaxed so that, at any time, a best link is selected from all available source-relay and relay-destination links. Depending on whether one $S-R$ or $R-D$ link is selected, either source or relay transmits opportunistically a data packet to the corresponding destination. The MLS schemes incorporate the instantaneous quality of wireless links as well as the states of finite relay buffers (e.g., full, empty, neither full nor empty) and adapt the time slot allocation accordingly. It was achieved that when all relay channels obey independent and identically distributed (i.i.d) Rayleigh fading, the MLS schemes not only obtain coding gain over the T-BRS and MMLS schemes but also has higher diversity gain than the two schemes. The achievable diversity order approaches two times of the one of TBRS and MMLS schemes in the ideal case where all channels experience i.i.d fading and the size of buffers grows to infinity. In [29], the buffer-aided amplifyand-forward (AF) MLS schemes have been considered over symmetric relaying channel. With the promising MLS protocol, in [30], a hybrid cooperative relaying policy was proposed. In [31], the buffer-aided multirelay cognitive system has been investigated.

From the above literature review, it is clearly seen that the introducing of buffer at relay can significantly improve the performance of the traditional cooperation communication systems. Especially, under the extreme case where all relay channels experience the i.i.d fading, the MLS schemes are promising since the higher diversity and coding gains can be achieved over the MMLS and T-BRS schemes. However, all of the existing works about the MLS buffer-aided relay cooperation only focused on the i.i.d fading channels. For the more general scenarios where all relay channels experience the independent and non-identically distributed (i.ni.d) fading, the corresponding performance of the MLS schemes has not been achieved so far. However, in practical wireless implementation, due to the fact that all relays can locate at different positions and the bad wireless propagation, the $S-R$ and $R-D$ relaying links can experience the nonidentical fading [32]. In this case, the weak link may 
have severe effect on the performance of the MLS schemes such as outage probability, symbol error rate, and average packet delay. Secondly, all of the existing works about the MLS schemes only consider Rayleigh fading scenario without Nakagami- $m$ one. It is well known that Nakagami- $m$ fading spans via the fading severity factor $m$, a wide range of fading scenarios that are typical in realistic wireless relay networks, which includes Rayleigh fading $(m=1)$ as special case. When the channels experience Nakagami- $m$ fading, the performance of the MLS schemes is impacted by not only the channel variances but also the fading severity factors.

Therefore, motivated by the above literature review, in this work, the buffer-aided MLS schemes are investigated over the i.ni.d Nakagami- $m$ fading channels. The contributions of this work are summarized as follows.

(1) By modeling the transition of buffer states as a Markov Chain, we first obtain the state transition matrix and the stationary state probability vector as well as the closed-form expressions in terms of outage probability and average packet delays over the i.ni.d Nakagami- $m$ fading channels. To the best of authors' knowledge, this is new. The presented comparison analysis shows clearly that when the relay channels experience the non-identical fading, the achievable diversity and coding gains by MLS schemes are suffered greatly from the disparity among the relay channel powers and the fading severity factors.

(2) For the average packet delays of MLS schemes, it is achieved that the average packet delays at all relays are the same entirely when the $S-R$ and $R-D$ links follow the i.i.d fading channels, respectively. Moreover, the average packet delays are also independent from the transmit power and the channel fading severity factors. However, under the i.ni.d fading channels, the average packet delays at all relays are different. This phenomenon yields that the MLS systems are unstable. At the same time, it is also achieved that when the powers of $S-R$ links are less than the ones of $R-D$ links, the average packet delays can be reduced evidently.

(3) In order to reduce the average packet delays and to enhance the stability of the MLS systems but still obtain the enough diversity and coding gains, we finally propose a weight-based MLS (W-MLS) diversitydelay trade-off scheme. By adjusting weight factor, it can be guaranteed that the equivalent signal-to-noise ratios (SNRs) of $S-R$ links are less than the ones of $R-D$ links such that the novel W-MLS schemes achieve the satisfying trade-off between diversity and delay.
Note that, for the MLS scheme, the work [26] is very important and inspired our work. For comparison in this work, we also employ the framework in [26]. However, the evident advancement is performed in our work over the work [26]. Firstly, our work focuses on the i.ni.d Nakagami- $m$ fading channels, while the work [26] considered the i.i.d Rayleigh ones. Secondly, our work investigates the impact of the i.ni.d fading channels on the diversity and coding gains as well as the average packet delay at relay. Finally, based on our derivations, we propose a weight-based MLS scheme, which can overcome the performance degradation caused by the i.ni.d fading.

Notation: In this work, we use boldface lower case letters to denote vectors and boldface capital letters to denote matrices. $\mathbf{O}_{m n}$ is a $m \times n$ matrix with all elements equal to zero, and I denotes the identity matrix. Moreover, (.) $)^{-1}$ and $(.)^{T}$ stand for matrix inversion and transpositions, respectively. $\mathbf{A}_{n m}$ is the $(n, m)$ th entry of $\mathbf{A} \cdot \operatorname{Pr}(X)$ denotes the probability of event $X . F_{X}($.$) and f_{X}($.$) are the cumulative distribu-$ tion function (CDF) and probability density function (PDF) of the random variable (RV) $X$, respectively. $\Gamma($.) and $\Upsilon(.,$.$) denote gamma function and incom-$ plete gamma function.

\section{System model and MLS schemes}

\subsection{System model and assumptions}

As shown in Fig. 1, we consider a buffer-aided relaying system, which consists of one source $S$, one destination $D, K$ relays $R_{k}, k \in\{1, \ldots, K\}$. Each relay node $R_{k}$ is equipped with a data buffer $Q_{k}$ of finite size $L$ (the number of buffer elements), and each buffer element can store one data packet of information bits. Note that, throughout this work, it is assumed that all buffers have the same size. Due to the severe fading and showing, we omit the direct link between source $S$ and destination $D$. All nodes work in FD mode, and there, they cannot transmit and receive

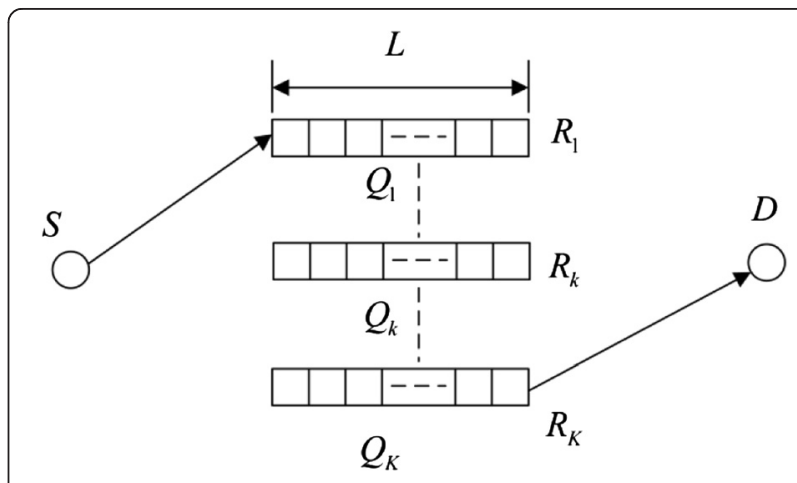

Fig. 1 Buffer-aided relaying system model 
simultaneously. We also define $\Psi\left(Q_{k}\right)$ denoting the number of data packets in buffer $Q_{k}$. We have $0 \leq \Psi\left(Q_{k}\right) \leq L$.

We assume that $h_{S R_{k}}$ denotes that channel coefficient between the source $S$ and the relay $R_{k}$ and $h_{R_{k} D}$ stands for the one between the relay $R_{k}$ and the destination $D$. The corresponding link gains are given by $\gamma_{S R_{k}}=\left|h_{S R_{k}}\right|^{2}$, $\gamma_{R_{k} D}=\left|h_{R_{k} D}\right|^{2}$. It is also assumed that all relay links are reciprocal and experience the i.ni.d Nakagami- $m$ fading. Therefore, the probability density function of the link gains $\gamma_{S R_{k}}$ and $\gamma_{R_{k} D}$ is given by

$$
f_{\gamma_{U}}(\gamma)=\frac{1}{\Gamma\left(m_{U}\right)}\left(\frac{m_{U}}{\omega_{U}}\right)^{m_{U}} \gamma^{m_{U^{-1}}} \exp \left(-\frac{m_{U}}{\omega_{U}} \gamma\right)
$$

where $U \in\left\{\gamma_{S R_{k}}, \gamma_{R_{k} D}\right\}, \omega_{U}$ and $m_{U}$ denote the corresponding channel variance and fading severity factor. At each receiver, the received signals are affected by the circularly symmetric complex Gaussian additive noise with identical noise power $N_{0}$. Without the loss of generality, throughout this work, we take the noise power $N_{0}=1$.

\subsection{MLS schemes}

In traditional best relay selection, the system performance is limited since in a round of communications, the same relay node is selected for receiving data from source and transmitting data to destination in a prefixed transmission order. Namely, in the T-BRS, both the available link quality and the degree of diversity freedom are not exploited effectively. Although the MMSL allows the different relays utilized for reception and transmission in a round of communications and exploit link quality, the scheme still follows a prefixed transmission order. Therefore, both the TBRS and MMSL schemes have the same diversity order. As an evolution of both the T-BRS and MMLS schemes, in the MLS schemes, the limitation on both the utilization of the same relay and the prefixed transmission order is relaxed so that, at any time, a best link is selected among all available $S-R$ and $R-D$ links. Therefore, the available diversity degree is exploited full, and under idea case, the diversity order is two times of the number of relays. With finite buffer size, a $S-R$ link is considered to be available when the corresponding relay node is not full and therefore can receive data packet from the source $S$, while a $R-D$ link is considered to be available when the relay node is not empty and thus can transmit stored data towards the destination $D$. The MLS scheme compares the quality of all the available links and adjusts the relay selection decision and the time slot allocation to the strongest link. If a $S-R$ link is the strongest link, the source $S$ transmits and the corresponding relay is selected for reception; on the other hand, if a $R-D$ link is the strongest link, the corresponding relay is selected for transmission. Therefore, from the above description, the MLS policy can be formulated as follows.

$$
b=\arg \max _{k}\left\{\underset{R_{k}: \Psi\left(Q_{k}\right) \neq L}{\cup}\left\{\gamma_{S R_{K}}\right\}, \underset{R_{k}: \Psi\left(Q_{k}\right) \neq 0}{\cup}\left\{\gamma_{R_{k} D}\right\}\right\}
$$

Due to the best link selected randomly, at a given time slot, the number $\Psi\left(Q_{k}\right)$ of data packets at relay $R_{k}$ is also random. Therefore, we define the random vector $\left\{\Psi\left(Q_{1}\right), \cdots, \Psi\left(Q_{K}\right)\right\}$ as a buffer state. Depending on the buffer's size $L$ and the number $K$ of relays, the total number of buffer states is given by $(L+1)^{K}$. Therefore, in this work, we assume that the vector $\mathbf{s}_{u}=\left\{\Psi_{u}\left(Q_{1}\right), \cdots, \Psi_{u}\left(Q_{K}\right)\right\}$ denotes the $u$ th buffer state, $1 \leq u \leq(L+1)^{K}$.

At the current state $\mathbf{s}_{u}$, the number $\Psi_{u}\left(Q_{k}\right)$ of data packets at the relay $R_{k}$ is increased by one when a source data packet is correctly decoded at $R_{k}$, while it is decreased by one when the relay $R_{k}$ successfully transmits a packet to the destination $D$. Depending on which relay receives or transmits data, the buffers may move from the current state $\mathbf{s}_{u}$ to several possible states. We model all possible states of buffers and the transitions between all sates as a Markov chain $(M C)$. The state transition diagram can be used to display MC directly, in which the connectivity between the different states of buffers is identified as follows: (a) the number of data elements of one relay buffer can be decreased by one if the relay node is selected for transmission and the transmission is successful; (b) the number of data elements of one relay buffer can be increased by one if the relay node is selected for receipting data and the reception is successful; and (c) the buffer state remains unchanged when the selected $S-R$ or $R-D$ transmission is not successful. At the same time, by using the state transition diagram, we can construct the corresponding state transition matrix A of $(L+1)^{K} \times(L+1)^{K}$, which is a square matrix. In the state transition matrix $\mathbf{A}$, the entries are zero when the corresponding state transition cannot be happened. The state transition matrix A is column stochastic, irreducible, and aperiodic. Therefore, the stationary state probability vector is given by

$$
\boldsymbol{\pi}=(\mathbf{A}-\mathbf{I}+\mathbf{B})^{-1} \mathbf{b}
$$


where $\pi=\left(\pi_{1}, \pi_{2}, \ldots \ldots, \pi_{(L+1)^{K}}\right)^{T}, \sum_{u=1}^{(L+1)^{K}} \pi_{u}=1, \mathbf{b}=$ $(1,1, \ldots . ., 1)^{T}$, $\mathbf{I}$ is identity matrix, and $\mathbf{B}_{n u}=1, \forall n, u$.

With the above formulating policy, for a bufferaided MLS system with $K=2$ and $L=2$, we have the state and the corresponding state transition diagram as shown in Fig. 2. In the state transition diagram, for a given sate $\mathbf{s}_{u}, \tilde{p}^{u}$ denotes the probability that the state $\mathbf{s}_{u}$ remains unchanged, and $p_{n u}$ denotes the probability of the transition from state $\mathbf{s}_{u}$ to $\mathbf{s}_{n}$, i.e., $p_{n u}=\operatorname{Pr}\left(\mathbf{s}_{u} \rightarrow \mathbf{s}_{n}\right)$. Since we assume that all relay channels experience the non-identically distributed fading, in general, we have $p_{n_{1} u} \neq p_{n_{2} u}$ for the state transition that can happen. On the other hand, for the one that cannot occur, there is no connectivity in the state diagram, and $p_{n u}=\operatorname{Pr}\left(\mathbf{s}_{u} \rightarrow \mathbf{s}_{n}\right)=0$. With the state transition diagram, the state transition matrix $\mathbf{A}$ can be achieved as well as the stationary state probability vector.

\section{Exact outage performance}

In this section, we investigate the exact outage performance of the buffer-aided MLS schemes. In MLS schemes, at state $\mathbf{s}_{u}$, if the selected $S-R$ or $R-D$ transmission is failed, there is one outage event. The probability that an outage event occurs depends on the available $S-R$ or $R-D$ links. Therefore, to obtain the outage probability, we define two sets $N_{S R}^{u}$ and $N_{R D}^{u}$ for state $\mathbf{s}_{u}$, where $N_{S R}^{u}$ contains the indices of all relays when the corresponding $S-R$ links are available for $S-R$ transmission at state $\mathbf{s}_{u}$ (the corresponding buffers are not full), and $N_{R D}^{u}$ contains the indices of all relays when the corresponding $R-D$ links are achievable for $R-D$ transmission (the corresponding buffers are not empty). The cardinalities of $N_{S R}^{u}$ and $N_{R D}^{u}$ are given by $\left|N_{S R}^{u}\right|$ and $\left|N_{R D}^{u}\right|$, respectively. Thus, for a given state $\mathbf{s}_{u}$, if there is one outage event, then no change in buffer state occurs. In state diagram, at the current state $\mathbf{s}_{u}$, the corresponding outage probability is given by $\tilde{p}^{u}$. In state transition matrix, the entries $\mathbf{A}_{u u}$ in the main diagonal stand for the outage probability at state $\mathbf{s}_{u}$. Obviously, we have $\tilde{p}^{u}=\mathbf{A}_{u u}$. By considering all possible states, the average outage probability of the systems is formulated as

$$
P_{\text {out }}=\sum_{u=1}^{(M+1)^{K}} \pi_{u} \mathbf{A}_{u u}=\sum_{u=1}^{(M+1)^{K}} \pi_{u} \tilde{p}^{u}
$$

In Eq.(4), the stationary distribution $\pi$ is given by Eq. (3). From Eq. (4), it is found that, to obtain the outage probability, the probability $\tilde{p}^{u}$ and the state transition matrix $\mathbf{A}$ are required.

\section{$3.1 \tilde{p}^{u}$ : outage probability at state $s_{u}$}

As stated previously, depending on which relay receives or transmits data, the buffers move from the current state $\mathbf{s}_{u}$ to several possible states. Therefore, we can divide all possible states to which can be moved from the current state $\mathbf{s}_{u}$ into two sets, $U_{S R}^{u-n}$ and $U_{R D}^{u-n}$, where $U_{S R}^{u-n}$ contains all states to which $\mathbf{s}_{u}$ can move when one $S-R$ link is selected and $U_{R D}^{u-n}$ contains all states to which $\mathbf{s}_{u}$ can move when one $R-D$ link is selected.

At the same time, from Eq. (2), it is observed that for the state $\mathbf{s}_{u}$ there are $\left|N_{S R}^{u}\right|$ and $\left|N_{R D}^{u}\right|$ terms in the first and second part maximizations within the "outer" max operation, respectively. Therefore, with Eq. (2), for state $\mathbf{s}_{u}$, we define the equivalent SNRs

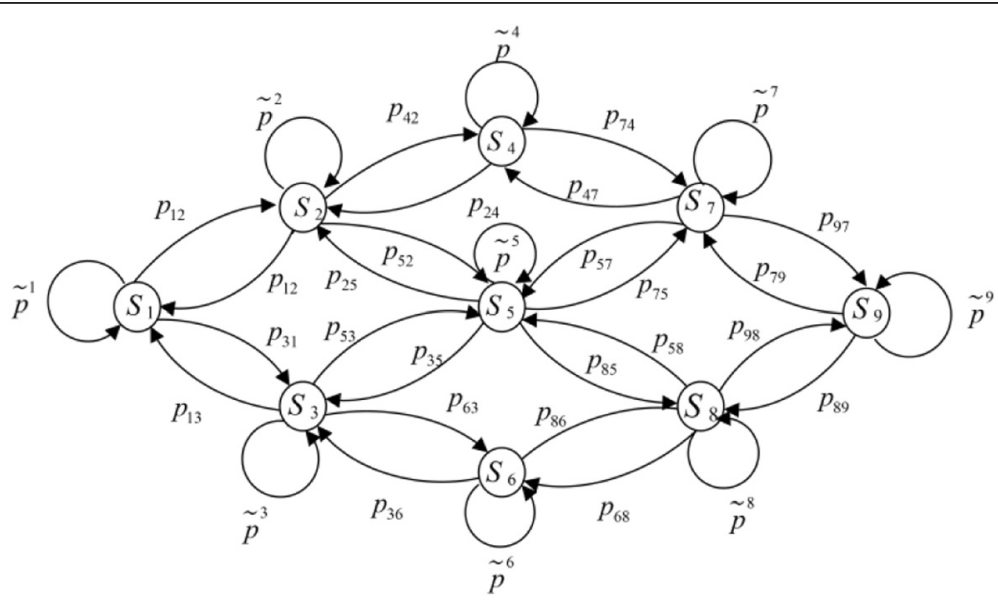

Fig. 2 State diagram for the MC of the MLS schemes with $K=2$ and $L=2$ 


$$
\gamma_{S R}^{u}=\max _{k \in N_{S R}^{u}}\left\{\gamma_{S R_{k}}\right\} \quad \gamma_{R D}^{u}=\max _{k \in N_{R D}^{u}}\left\{\gamma_{R_{k} D}\right\}
$$

Then, we can utilize $\gamma_{\text {end }}^{u}=\max \left\{\gamma_{S R}^{u}, \gamma_{R D}^{u}\right\}$ to complete the max-link selection for the overall system. The outage probability corresponding to the pair $\left(N_{S R}^{u}, N_{R D}^{u}\right)$ for the state $\mathbf{s}_{u}$ is given by

$$
\tilde{p}^{u}=\operatorname{Pr}\left\{\gamma_{S R}^{u} \leq \gamma_{t h}\right\} \times \operatorname{Pr}\left\{\gamma_{R D}^{u} \leq \gamma_{t h}\right\}
$$

where $\gamma_{t h}$ is the predefined outage threshold which is determined by the spectral efficiency, transmission power $P$, and additive noise power $N_{0}$. With the consideration that all relay channels obey the i.ni.d Nakagami- $m$ fading, using Eq. (1) leads to the first term in Eq. (6) written as

$$
\begin{aligned}
\operatorname{Pr}\left\{\gamma_{S R}^{u} \leq \gamma_{t h}\right\} & =\operatorname{Pr}\left\{\max _{k \in N_{S R}^{u}}\left\{\gamma_{S R_{k}}\right\} \leq \gamma_{t h}\right\} \\
& =\prod_{k=1}^{\left|N_{S R}^{u}\right|} \frac{1}{\Gamma\left(m_{S R_{S_{S R}^{u}}^{u}(k)}\right)} \gamma\left(m_{S R_{N_{S R}^{u}(k)}}, \frac{m_{S R_{N_{S R}^{u}(k)}}}{\omega_{S R_{N_{S R}^{u}}(k)}} \gamma_{t h}\right)
\end{aligned}
$$

With similar reason, the second term in Eq. (6) is written as

$$
\begin{aligned}
& \operatorname{Pr}\left\{\gamma_{R D}^{u} \leq \gamma_{t h}\right\} \\
& \quad=\prod_{k=1}^{\left|N_{R D}^{u}\right|} \frac{1}{\Gamma\left(m_{R_{N_{R D}^{u}(k)} D}\right)} \gamma\left(m_{R_{N_{R D}^{u}(k)} D}, \frac{m_{R_{R_{R D}^{u}}^{u}(k)} D}{\omega_{R_{N_{R D}^{u}(k)} D}} \gamma_{t h}\right)
\end{aligned}
$$

Finally, the outage probability $\tilde{p}^{u}$ at the state $\mathbf{s}_{u}$ can be achieved by substituting Eqs. (7) and (8) into Eq. (6).

\section{2 $\mathrm{A}_{n u}$ : the $(n, u)$ th entry of transition matrix $\mathrm{A}$}

Besides $\tilde{p}^{u}$, to obtain the total outage probability, Eq. (4) indicates that the stationary distribution $\pi$ is also required. At the same time, the Eq. (3) shows that the stationary distribution $\pi$ depends on the transition matrix A. Because all relay links exhibit non-identical fading, at any time, the probabilities to select the $S-R$ and $R-D$ transmissions are not the same. This is very different from the existing buffer-aided MLS schemes where all relaying links exhibit identical distribution and the selection of any available link is equally likely. With this observation, we define $p_{S R}^{u}$ and $p_{R D}^{u}$ as the probabilities that the $S-R$ and $R-D$ transmissions are selected at state $\mathbf{s}_{u}$, respectively. It is clear that we have $p_{S R}^{u}+p_{R D}^{u}=1$. We also define $p_{N_{S R}^{u}}^{n}$ as the probability that the best $S-R$ link is selected from the available $\left|N_{S R}^{u}\right| S-R$ links such that the state transition $\mathbf{s}_{u}->\mathbf{s}_{n}$ occurs and $p_{N_{R D}^{n}}^{n}$ is the probability that the best $R-D$ link is selected from the available $\left|N_{R D}^{u}\right| R-D$ links such that the state transition $\mathbf{s}_{u}->\mathbf{s}_{n}$ occurs. Therefore, the probabilities to select one $S-R$ link and one $R-D$ link at state $\mathbf{s}_{u}$ are formulated, respectively, as

$$
\begin{aligned}
& p_{S R}^{(u, n)}=p_{N_{S R}^{u}}^{n} \times p_{S R}^{u} ; \\
& p_{R D}^{(u, n)}=p_{N_{R D}^{u}}^{n} \times p_{R D}^{u}=p_{N_{R D}^{u}}^{n} \times\left(1-p_{S R}^{u}\right)
\end{aligned}
$$

With these observations, the $\mathbf{A}_{n u}$ entry of the state transition matrix $\mathbf{A}$ is expressed as

$$
\mathbf{A}_{n u}=\left\{\begin{array}{lc}
\tilde{p}^{u} & \text { if } n=u \\
p_{N_{S R}^{u}}^{n} \times p_{S R}^{u} \times\left(1-\tilde{p}^{u}\right) & \text { if } n \neq u \text { and } n \in U_{S R}^{u-n} \\
p_{N_{R D}^{u}}^{u} \times\left(1-p_{S R}^{u}\right) \times\left(1-\tilde{p}^{u}\right) & \text { if } n \neq u \text { and } n \in U_{R D}^{u-n} \\
0 & \text { elsewhere }
\end{array}\right.
$$

3.3 $p_{S R}^{u}$ : probability that the $S-R$ transmission is selected at state $s_{u}$

At state $\mathbf{s}_{u}$, if there is no $S-R$ link available (i.e., $\left|N_{S R}^{u}\right|=0$ ), we have the probability $p_{S R}^{u}=0$. On the other hand, if there is no $R-D$ link available (i.e., $\left|N_{R D}^{u}\right|=0$ ), $p_{S R}^{u}=1$. For other case (i.e., $\left|N_{S R}^{u}\right| \neq 0$ and $\left|N_{R D}^{u}\right| \neq 0$ ), the probability $p_{S R}^{u}$ is formulated as

$$
p_{S R}^{u}=\operatorname{Pr}\left\{\gamma_{S R}^{u} \geq \gamma_{R D}^{u}\right\}
$$

where $\gamma_{S R}^{u}$ and $\gamma_{R D}^{u}$ are defined by Eq. (5). With order statistics [33], the Eq. (11) can be rewritten as

$$
\begin{aligned}
p_{S R}^{u} & =\int_{0}^{\infty} \operatorname{Pr}\left\{\gamma_{S R}^{u} \geq y\right\} f_{\gamma_{R D}^{u}}(y) d y \\
& =1-\int_{0}^{\infty} \operatorname{Pr}\left\{\gamma_{S R}^{u}<y\right\} f_{\gamma_{R D}^{u}}(y) d y
\end{aligned}
$$

where $f_{\gamma_{R D}^{u}}(y)$ is the probability density function of the $\mathrm{RV} \gamma_{R D}^{u}=\max _{k \in N_{R D}^{u}}\left\{\gamma_{R_{k} D}\right\}$. By using order statistics [33], after appropriate mathematic manipulation, the probability $p_{S R}^{u}$ is given by 


$$
p_{S R}^{u}=1-\sum_{1} \sum_{2} \Gamma\left(\sum_{t=1}^{k} h_{t}+\sum_{t=1}^{f} v_{t}+m_{R_{N_{R D}^{u}(k)} D}\right)\left(\sum_{t=1}^{k} \frac{m_{S R_{N_{S R}^{u}\left(t_{t}\right)}}}{\omega_{S R_{N_{S R}^{u}\left(q_{t}\right)}}}+\sum_{t=1}^{f} \frac{m_{R_{N_{R D}^{u}}\left(t_{t}\right)^{D}}}{\omega_{R_{N_{R D}^{u}}\left(q_{t}\right)} D} y+\frac{m_{R_{N_{R D}^{u}(k)} D}}{\omega_{R_{N_{R D}^{u}(k)} D}}\right)^{-\left(\sum_{t=1}^{k} h_{t}+\sum_{t=1}^{f} v_{t}+m_{R_{R D}^{u}(k)}\right)^{D}}
$$

where $\Sigma_{1} \Sigma_{2}$ are defined, respectively, by

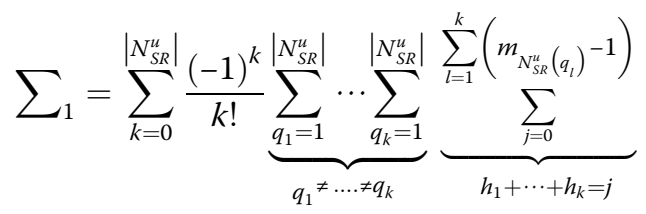

$$
\begin{aligned}
& \prod_{t=1}^{k} \frac{1}{\left(h_{t}\right) !}\left(\frac{m_{N_{S R}^{u}\left(q_{t}\right)}}{\left.\omega_{N_{S R}^{u}\left(q_{t}\right)}\right)}\right)^{h_{t}} \\
& \sum_{2}=\sum_{k=1}^{\left|N_{R D}^{u}\right|} \frac{1}{\Gamma\left(m_{R_{N_{S R}^{u}(k)}^{u} D}\right)}\left(\frac{m_{R_{N_{D D}^{u}(k)} D}}{\omega_{R_{N_{R D}(k)} D}}\right)^{m_{R_{N} u}^{u}(k)} \sum_{f=0}^{D} \frac{N_{R D}^{u}|-1|}{f !}
\end{aligned}
$$

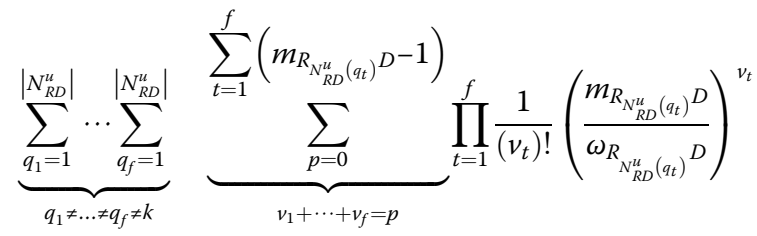

The proof of Eq. (13) is presented in Appendix 1.

$3.4 p_{N_{S R}^{u}}^{n}$ and $p_{N_{R D}^{u}}^{n}$

We know that $p_{N_{S R}^{u}}^{n}$ is the probability that the best $S-R$ link is selected from all available $\left|N_{S R}^{u}\right| S-R$ links such that the state transition $\mathbf{s}_{u}->\mathbf{s}_{n}$ occurs and $p_{N_{R D}^{u}}^{n}$ is the probability that the best $R-D$ link is selected from all available $\left|N_{R D}^{u}\right| R-D$ links. Moreover, at the current state $\mathbf{s}_{u}$, for given state transition $\mathbf{s}_{u}->\mathbf{s}_{n}$, the selected $S-R$ or $R-D$ link is determined, which can be obtained from state transition table. To illustrate this consideration, in Table 1, we present the selected $S-R$ or $R-D$ link for each state transition $\mathbf{s}_{u}->\mathbf{s}_{n}$, where we take $K=2$ and $L=2$. We have the state $\mathbf{s}_{u}=\left\{\Psi_{u}\left(Q_{1}\right), \Psi_{u}\left(Q_{2}\right)\right\}$. Table 1 shows clearly that, for each given state transition $\mathbf{s}_{u}->\mathbf{s}_{n}$, the selected $S-R$ or $R-D$ link is determined. For example, when the state transition $11->10$ occurs, the $R_{2} D$ link is selected for data transmission.
Therefore, with the above observation, without loss of generality, we assume that the ath element in $N_{S R}^{u}$ or $N_{R D}^{u}$ is selected so that the state transition $\mathbf{s}_{u}->\mathbf{s}_{n}$ arises. We first consider $p_{N_{S R}^{u}}^{n}$. Since there is only one possible link is selected, the probability $p_{N_{S R}^{u}}^{n}$ can be written as [33]

$$
p_{N_{S R}^{u}}^{n}=\operatorname{Pr}\left\{\gamma_{S R_{N_{S R}^{u}(a)}} \geq Y_{N_{S R}^{u}(a)}\right\}
$$

where the RV $Y_{N_{S R}^{u}(a)}$ is defined as $Y_{N_{S R}^{u}(a)}=\max _{k \in N_{S R}^{u}, k \neq a}\left\{\gamma_{S R_{N_{S R}^{u}}^{u}(k)}\right\}$, $a$ is the function of $u$ and $n$. Therefore, by using order statistics [33], after appropriate mathematic manipulation, the probability $p_{N_{S R}^{u}}^{n}$ is given by [34]

$$
\begin{aligned}
p_{N_{S R}^{u}}^{n}=\sum_{3} \Gamma & \left(\sum_{t=1}^{i} g_{t}+m_{S R_{N_{S R}^{u}(a)}}\right) \\
& \left(\sum_{t=1}^{i} \frac{m_{S R_{N_{S R}^{u}\left(f_{t}\right)}}}{\omega_{S R_{N_{S R}^{u}(t)}(t)}}+\frac{m_{S R_{N_{S R}^{u}}^{u}(a)}}{\omega_{S R_{N_{S R}^{u}}^{u}(a)}}\right)-\left(\sum_{t=1}^{i} g_{\left.t^{+}+m_{S R_{N R}^{u}(a)}\right)}\right)
\end{aligned}
$$

where $\Sigma_{3}$ is defined by

$$
\begin{aligned}
& \sum_{3}=\frac{1}{\Gamma\left(m_{S R_{N_{S R}}^{u}(a)}\right)}\left(\frac{m_{S R_{S_{S R}^{u}}(a)}}{\omega_{S R_{N_{S R}^{u}}^{u}(a)}}\right)^{m_{S R_{N R}^{u}}^{u}(a)} \sum_{i=0}^{\left|N_{S R R}^{u}\right|-1} \frac{(-1)^{i}}{i !} \\
& \underbrace{\sum_{f_{1}=1}^{\left|N_{S R}^{u}\right|} \cdots \sum_{f_{i}=1}^{\left|N_{S R}^{u}\right|}}_{f_{1} \neq \ldots \neq f_{i} \neq a} \underbrace{\sum_{t=1}^{i}\left(m_{S R N_{S R}^{m}\left(f_{t}\right)^{-1}} \sum_{j=1}\right.}_{g_{1}+\cdots+g_{k}=j} \prod_{t=1}^{i} \frac{1}{\left(g_{t}\right) !}\left(\frac{\left.m_{S R N_{S R}^{u}\left(f_{t}\right)}\right)^{g_{S R N_{S R}^{u}}\left(f_{t}\right)}}{g_{t}}\right.
\end{aligned}
$$

The proof of Eq. (17) can be found in Appendix 2. Similar to Eq. (17), the closed-form expression of $p_{N_{R D}^{u}}^{n}$ can be achieved, which has the similar form as Eq. (17). 
Table 1 The selected $S-R$ or $R-D$ link for the state transition from $\mathbf{s}_{u}$ to $\mathbf{s}_{n}\left(\mathbf{s}_{u}=\left\{\psi_{u}\left(Q_{1}\right), \psi_{u}\left(Q_{2}\right)\right\}\right)$

\begin{tabular}{|c|c|c|c|c|c|c|c|c|c|}
\hline & $\mathbf{s}_{u}: 00$ & $\mathbf{s}_{u}: 01$ & $\mathbf{s}_{u}: 02$ & $\mathbf{s}_{u}: 10$ & $\mathbf{s}_{u}: 11$ & $\mathbf{s}_{u}: 12$ & $\mathbf{s}_{u}: 20$ & $\mathbf{s}_{u}: 21$ & $\mathbf{s}_{u}: 22$ \\
\hline $\mathbf{s}_{n}: 00$ & & $R_{2} D$ & & $R_{1} D$ & & & & & \\
\hline $\mathbf{s}_{n}: 01$ & $S R_{2}$ & & $R_{2} D$ & & $R_{1} D$ & & & & \\
\hline $\mathbf{s}_{n}: 02$ & & $S R_{2}$ & & & & $R_{1} D$ & & & \\
\hline $\mathbf{s}_{n}: 10$ & $S R_{1}$ & & & & $R_{2} D$ & & $R_{1} D$ & & \\
\hline $\mathbf{s}_{n}: 11$ & & $S R_{1}$ & & $S R_{2}$ & & $R_{2} D$ & & $R_{1} D$ & \\
\hline $\mathbf{s}_{n}: 12$ & & & $S R_{1}$ & & $S R_{2}$ & & & & $R_{1} D$ \\
\hline $\mathbf{s}_{n}: 20$ & & & & $S R_{1}$ & & & & $R_{2} D$ & \\
\hline $\mathbf{s}_{n}: 21$ & & & & & $S R_{1}$ & & $S R_{2}$ & & $R_{2} D$ \\
\hline $\mathbf{s}_{n}: 22$ & & & & & & $S R_{1}$ & & $S R_{2}$ & \\
\hline
\end{tabular}

Therefore, by substituting $p_{N_{R D}^{u}}^{n}$, Eq. (17), and Eq. (13) into Eq. (10), we can obtain A. Then, using Eq. (3) leads to the stationary state probability vector $\pi$. Finally, substituting $\pi$ and $\tilde{p}^{u}$ given by Eq. (6) into Eq. (4), the outage probability is achieved.

\section{Asymptotic performance analysis}

In previous sections, we obtain the outage probability of the buffer-aided MLS schemes over the i.ni.d Nakagami- $m$ fading channels. To highlight insight, in this section, we consider some special cases, i.e., asymmetric relay channels and symmetric relay channels. We mainly discuss the probabilities $p_{S R}^{u}, p_{N_{S R}^{u}}^{u}$, and $p_{N_{R D}^{u}}^{n}$.

\subsection{Identically distributed and asymmetric relay channels} When the identically distributed and asymmetric relay channels are considered, we have $m_{S R_{k}}=m_{S R}, \omega_{S R_{k}}=\omega_{S R}$, and $m_{R_{k} D}=m_{R D}, \omega_{R_{k} D}=\omega_{R D}$, but $m_{S R} \neq m_{R D}, \omega_{S R} \neq \omega_{R D}$. For such case, we first consider the case where $\left|\omega_{S R}-\omega_{R D}\right|$ is little, relatively. Due to the fact that the numbers of available links for $S-R$ and $R-D$ transmissions at state $\mathbf{s}_{u}$ are $\left|N_{S R}^{u}\right|$ and $\left|N_{R D}^{u}\right|$, respectively, using the results in [35] and [36] leads to the probability $p_{S R}^{u}$ in Eq. (12) is written as where $\left(\begin{array}{l}N \\ n\end{array}\right)=\frac{N !}{n !(N-n) !}$ is binomial coefficient. For a more tractable form, using the multinomial expansion theorem, in Eq. (19), the first multinomial is written as

$$
\begin{aligned}
& \left(\sum_{n_{1}=0}^{m_{S R}-1} \frac{1}{n_{1} !}\left(\frac{m_{S R}}{\omega_{S R}} y\right)^{n_{1}}\right)^{m_{1}}=\sum_{a_{0}+\cdots+a_{m_{S R}-1}=m_{1}}\left(\begin{array}{c}
m_{1} \\
a_{0}, a_{1}, \cdots a_{m_{S R}-1}
\end{array}\right) \\
& \prod_{0 \leq t \leq m_{S R}-1}\left(\frac{1}{t !}\right)^{a_{t}}\left(\frac{m_{S R}}{\omega_{S R}} y\right) \sum_{t=0}^{m_{S R}-1} t \cdot a_{t}
\end{aligned}
$$

where $\left(\begin{array}{c}m_{1} \\ a_{0}, a_{1}, \cdots a_{m_{S R}-1}\end{array}\right)=\left(\frac{m_{1} !}{a_{0} ! \cdots\left(a_{m_{S R^{-1}}}\right) !}\right)$ denotes the multinomial coefficient. Similarly, in Eq. (19), the second multinomial is written as

$$
\begin{aligned}
\left(\sum_{n_{2}=0}^{m_{R D}-1} \frac{1}{n_{2} !}\left(\frac{m_{R D}}{\omega_{R D}} y\right)^{n_{2}}\right)^{m_{2}} & =\sum_{b_{0}+\cdots+b_{m_{R D}-1}=m_{2}}\left(\begin{array}{c}
m_{2} \\
b_{0}, b_{1}, \cdots b_{m_{R D}-1}
\end{array}\right) \\
& \prod_{0 \leq t \leq m_{R D}-1}\left(\frac{1}{t !}\right)^{b_{t}}\left(\frac{m_{R D}}{\omega_{R D}} y\right) \sum_{t=0}^{m_{R D} D^{-1} t \cdot b_{t}}
\end{aligned}
$$

Combing Eqs. (21), (20), and (19), we have the following integral.

$$
\begin{aligned}
& p_{S R}^{u}=1-\frac{\left|N_{R D}^{u}\right|}{\Gamma\left(m_{R D}\right)}\left(\frac{m_{R D}}{\omega_{R D}}\right)^{m_{R D}} \times \int_{0}^{\infty} y^{m_{R D}-1} e^{-\frac{m_{R D}}{\omega_{R D}} y}
\end{aligned}
$$

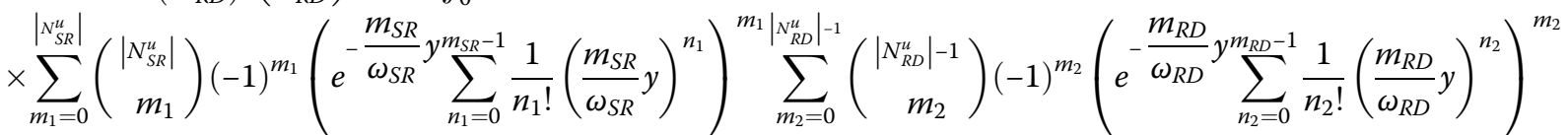




$$
\begin{aligned}
& \int_{0}^{\infty} \sum^{m_{1}=0} t_{1} a_{t_{1}}+\sum_{t_{2}=0}^{m_{R D}-1} t_{2} a_{t_{2}+m_{R D}-1} \cdot e^{-y\left(\frac{m_{R D}}{\omega_{R D}} \times m_{2}+\frac{m_{S R}}{\omega_{S R}} \times m_{1}+\frac{m_{R D}}{\omega_{R D}}\right)} d y \\
& =\Gamma\left(\sum_{t_{1}=0}^{m_{S R}-1} t_{1} a_{t_{1}}+\sum_{t_{2}=0}^{m_{R D}-1} t_{2} a_{t_{2}}+m_{R D}\right)\left(\frac{m_{R D}}{\omega_{R D}} \times\left(m_{2}+1\right)+\frac{m_{S R}}{\omega_{S R}} \times m_{1}\right)
\end{aligned}
$$

Therefore, for the identically distributed and asymmetry case, the probability of the $S-R$ transmission selected is

$$
\begin{aligned}
& p_{S R}^{u}=1-\frac{\left|N_{R D}^{u}\right|}{\Gamma\left(m_{R D}\right)}\left(\frac{m_{R D}}{\omega_{R D}}\right)^{m_{R D}} \sum_{m_{1}=0}^{\left|N_{S R}^{u}\right|\left|N_{R D}^{u}\right|-1} \sum_{m_{2}=0} \sum_{a_{0}+\cdots+a_{m_{S R}-1}=m_{1}} \sum_{b_{0}+\cdots+b_{m_{R D}-1}=m_{2}}(-1)^{m_{1}+m_{2}}\left(\begin{array}{c}
\left|N_{S R}^{m}\right| \\
m_{1}
\end{array}\right)\left(\begin{array}{c}
\left|N_{R D}^{m}\right|-1 \\
m_{2}
\end{array}\right) \\
& \times\left(\begin{array}{c}
m_{1} \\
a_{0}, \cdots a_{m_{S R}-1}
\end{array}\right)\left(\begin{array}{c}
m_{2} \\
a_{0}, \cdots a_{m_{R D}-1}
\end{array}\right)\left(\frac{m_{S R}}{\omega_{S R}}\right)^{\sum_{t_{1}=0}^{m_{S R}-1} t_{1} \cdot a_{t_{1}}}\left(\frac{m_{R D}}{\omega_{R D}}\right)^{\sum_{t_{2}=0}^{m_{R D}-1} t_{2} \cdot a_{t_{2}}} \prod_{0 \leq t_{1} \leq m_{S R}-1}\left(\frac{1}{t_{1} !}\right)^{a_{t_{1}}} \prod_{0 \leq t_{2} \leq m_{R D}-1}\left(\frac{1}{t_{2} !}\right)^{a_{t_{2}}} \\
& \times \Gamma\left(\sum_{t_{1}=0}^{m_{S R}-1} t_{1} a_{t_{1}}+\sum_{t_{2}=0}^{m_{R D}-1} t_{2} a_{t_{2}}+m_{R D}\right)\left(\frac{m_{R D}}{\omega_{R D}} \times\left(m_{2}+1\right)+\frac{m_{S R}}{\omega_{S R}} \times m_{1}\right)^{-\left(\sum_{t_{1}=0}^{m_{S R}-1} t_{1} a_{t_{1}}+\sum_{t_{2}=0}^{m_{R D}-1} t_{2} a_{t_{2}}+m_{R D}\right)}
\end{aligned}
$$

With Eq. (23), having $p_{R D}^{m}=1-p_{S R}^{m}$.

For $p_{N_{S R}^{u}}^{n}$, according to Eq. (17), when the all $S-R$ channels are i.i.d fading, the probability $p_{N_{S R}^{u}}^{n}$ to select one $S-R$ link from all available $\left|N_{S R}^{u}\right| S-R$ links is given by

$$
\begin{aligned}
p_{N_{S R}^{u}}^{n}= & \int_{0}^{\infty}\left[1-\frac{1}{\Gamma\left(m_{S R}\right)} \Gamma\left(m_{S R}, \frac{m_{S R}}{\omega_{S R}} y\right)\right]^{\left|N_{S R}^{u}\right|-1} \\
& \frac{1}{\Gamma\left(m_{S R}\right)}\left(\frac{m_{S R}}{\omega_{S R}}\right)^{m_{S R}} y^{m_{S R}-1} e^{-\frac{m_{S R}}{\omega_{S R}}} d y=\frac{1}{\left|N_{S R}^{u}\right|}
\end{aligned}
$$

Similarly, when all $R-D$ channels are i.i.d fading, the probability $p_{N_{R D}^{u}}^{n}$ to select a $R-D$ link is given by

$$
p_{N_{R D}^{u}}^{n}=\frac{1}{\left|N_{R D}^{u}\right|}
$$

By substituting Eqs. (23), (24), and (25) into Eq. (10), we can obtain the state transition matrix $\mathbf{A}$ as well as the resulting stationary state probability vector $\pi$ from Eq. (3) for the identically distributed and asymmetric relay channels.

4.2 Symmetric case $\left(\omega_{S R_{k}}=\omega_{R_{k} D}=\omega, \quad m_{S R_{k}}=m_{R_{k} D}=m\right)$ For the symmetric case (or i.i.d case), we have $m_{S R_{k}}$ $=m_{R_{k} D}=m \quad$ and $\quad \omega_{S R_{k}}=\omega_{R_{k} D}=\omega, k \in\{1, \ldots, K\}$.
Therefore, the probability $p_{S R}^{m}$ that the $S-R$ transmission is selected is given by

$$
\begin{aligned}
P_{S R}^{u}= & 1-\left|N_{R D}^{u}\right| \int_{0}^{\infty}\left[1-\frac{1}{\Gamma(m)} \Gamma\left(m, \frac{m}{\omega}\right)\right]^{\left|N_{S R}^{u}\right|+\left|N_{R D}^{u}\right|-1} \\
& \frac{1}{\Gamma(m)} \Gamma\left(\frac{m}{\omega}\right)^{m} y^{m-1} e^{-\frac{m}{\omega} y} d y=\frac{\left|\mathrm{N}_{\mathrm{SR}}^{u}\right|}{\left|\mathrm{N}_{\mathrm{SR}}^{u}\right|+\left|\mathrm{N}_{\mathrm{RD}}^{u}\right|}
\end{aligned}
$$

The probability $p_{R D}^{m}$ to select $R-D$ transmission at state $\mathbf{s}_{m}$ is

$$
p_{R D}^{u}=1-p_{S R}^{u}=\frac{\left|N_{R D}^{u}\right|}{\left|N_{R D}^{u}\right|+\left|N_{S R}^{u}\right|}
$$

With Eq. (24), in Eq. (9), we have

$$
\begin{aligned}
& p_{S R}^{(u, n)}=p_{N_{S R}^{u}}^{u} \times p_{S R}^{u}=\frac{1}{\left|N_{R D}^{u}\right|+\left|N_{S R}^{u}\right|}, \\
& p_{R D}^{(u, n)}=p_{N_{R D}^{u}}^{n} \times p_{R D}^{u}=\frac{1}{\left|N_{R D}^{u}\right|+\left|N_{S R}^{u}\right|}
\end{aligned}
$$

\section{Diversity and coding gains}

In this section, we consider the diversity and coding gains. To this end, in Eq. (6), we denote $\gamma_{t h}=\frac{\gamma_{t h}{ }^{\prime}}{P}$, where $\gamma_{t h}^{\prime}$ is determined by the spectrum efficiency. It is well 
known, when $x \rightarrow 0$, the incomplete gamma function $r$ $(m, x) \sim \frac{\chi^{m}}{m}$. Therefore, at state $\mathbf{s}_{u}$, the probability $\tilde{p}^{u}$ is written as

$$
\begin{aligned}
& \tilde{p}^{u} \approx \prod_{\mathrm{k}=1}^{\left|N_{S R}^{m}\right|} \frac{1}{\Gamma\left(m_{S R_{N_{S R} m}(k)}+1\right)}\left(\frac{m_{S R_{N_{S R}^{\prime m}}(k)}}{\omega_{S R_{N_{S R}^{m}}(k)}} \frac{\gamma_{t h}{ }^{\prime}}{P}\right)^{m_{S R_{N_{S R} m}(k)}} \\
& \prod_{\mathrm{k}=1}^{\left|N_{R D}^{m}\right|} \frac{1}{\Gamma\left(m_{R_{N_{S R}^{m}(k)} D}+1\right)}\left(\frac{m_{R_{N_{S R}^{m}(k)} D}}{\omega_{R_{N_{S R}^{m}}(k)} D} \frac{\gamma_{t h}{ }^{\prime}}{P}\right)^{m_{R_{N R} m}(k){ }^{D}}
\end{aligned}
$$

Then, with $P_{\text {out }}=\sum_{u=1}^{(M+1)^{K}} \pi_{u} \tilde{p}^{u}$, we can obtain the diversity order

$$
G_{d}=\lim _{p \rightarrow \infty}\left(-\frac{\log p_{\text {out }}}{\log \left(\frac{P}{\gamma_{\text {th }}}\right)}\right)
$$

However, Eqs. (29) and (30) show that it is challenging to achieve the diversity order due to the i.ni.d assumption. Therefore, we resort to the asymptotic analysis for the diversity and coding gains. We consider the identically distributed and asymmetric fading channels, $\omega_{S R_{k}}=\omega_{S R}, \omega_{R_{k} D}=\omega_{R D}, \omega_{S R} \neq \omega_{R D}, m_{S R_{k}}=m_{R_{k} D}=m$. With this assumption, Eq. (29) is given by

$$
\begin{aligned}
\tilde{p}^{u} \approx & \left(\frac{\gamma_{t h}{ }^{\prime}}{P}\right)^{\left(\left|N_{S R}^{u}\right|+\left|N_{R D}^{u}\right|\right) m}\left(\frac{1}{\Gamma(m+1)}\left(\frac{m}{\omega_{S R}}\right)^{m}\right)^{\left|N_{S R}^{u}\right|} \\
& \left(\frac{1}{\Gamma(m+1)}\left(\frac{m}{\omega_{R D}}\right)^{m}\right)^{\left|N_{R D}^{u}\right|}
\end{aligned}
$$

With the consideration that the distribution of $\left(N_{S R}^{m}, N_{R D}^{m}\right)$ depends on both the number of relays $K$ and the relay buffer size $L$, we consider the following two special cases. Firstly, when the buffer size $L=1$, we always have $\left|N_{S R}^{m}\right|+\left|N_{R D}^{m}\right|=K$. The outage probability is written as

$$
\begin{aligned}
P_{\text {out }}= & \left(\frac{\gamma_{\text {th }}{ }^{\prime}}{P}\right)^{K m} \Delta_{A} \\
\Delta_{A}= & \sum_{u=1}^{(M+1)^{K}} \pi_{u}\left(\frac{1}{\Gamma(m+1)}\left(\frac{m}{\omega_{S R}}\right)^{m}\right)^{\left|N_{S R}^{u}\right|} \\
& \left(\frac{1}{\Gamma(m+1)}\left(\frac{m}{\omega_{R D}}\right)^{m}\right)^{\left|N_{R D}^{u}\right|}
\end{aligned}
$$

At the same time, from Eq. (23)-(25), we see that in this case, $\pi_{u}$ is independent from $\gamma$. Therefore, substituting Eq. (32) into (30), we have that the diversity order is

$$
G_{d}=\lim _{P \rightarrow \infty}\left(-\frac{\log p_{\text {out }}}{\log \left(\frac{P}{\gamma_{t h}{ }^{\prime}}\right)}\right)=m K
$$

which is the lower bound. On the other hand, when the relay buffer size $L->\infty$, it can be shown that the probabilities for $\left|N_{S R}^{m}\right|=K$ and $\left|N_{R D}^{m}\right|=K$ are one. Correspondingly, the max link is selected from $2 K$ available links, and $\left|N_{S R}^{m}\right|+\left|N_{R D}^{m}\right|=2 K$. The diversity order is

$$
G_{d}=2 m K
$$

The result shows that the diversity order is close to $2 m K$, which is upper bound. In general, the diversity order of the considered scheme is between $m K$ and $2 m K$ and increasing with the buffer size $L$ due to the asymmetry. With the diversity order, the coding gain is written as

$$
G_{c}=\frac{\left(\Delta_{A}\right)^{-\frac{1}{G_{d}}}}{\gamma_{t h}^{\prime}}
$$

\section{Average packet delay}

The use of buffers at the relays improves the performance at the expense of a higher packet delay in systems. The delay is a main issue of the buffer-aided relaying systems. Especially, the packet delay problem is becoming more severe in MLS schemes. This is because that in MLS schemes, the best link is selected from all available $S-R$ and $R-D$ links. In this case, the weak links may have severe effect on the buffer-aided MLS relay systems. For example, we consider a MLS system with two relays, where the channel average powers of $S-R$ and $R-D$ links are $\{10,1\}$ and $\{0.01,1\}$, respectively. Obviously, the MLS protocol fills the buffer of the first relay very soon. Since the link of the first relay to destination has a very bad quality, the first relay will not contribute to the performance of the systems. However, the data packets in the buffer of the first relay will be trapped for a long time. This will eventually yields a higher delay of the data packets. Hence, it is important to study the end-toend delay to understand the performance-delay trade-off of the buffer-aided MLS relaying schemes. In this section, we analyze the average packet delay of the MLS systems. We provide a closed-form expression for the average packet delay in terms of the buffer size and the probabilities of the link selection. We particularly highlight that, while different packets may suffer from different delay, the system throughput (or the average data packet rate) of the MLS schemes is not scarified. This is 
because that in each time slot, a relay node must be selected for receiving or forwarding data packet. Therefore, without losing the generality, in sequent, we assume that the average throughput is 1 (packet/slot). Therefore, when a packet is "waiting" for transmission at a node, another packet must be transmitted to another node. According to Little's law, the average packet delay at a given relay node can be formulated as

$$
E\left\{D_{k}\right\}=\frac{E\left\{\Psi\left(Q_{k}\right)\right\}}{\eta_{k}}, \quad k \in\{1, \ldots, K\}
$$

where $E\left\{\Psi\left(Q_{k}\right)\right\}$ and $\eta_{k}$ are the average queuing packet length and throughput at relay node $R_{k}$. Therefore, the average arrival rate into the buffer of the relay $R_{k}$ is given by

$$
\eta_{k}=\sum_{u=1}^{(L+1)^{K}} \pi_{u} p_{S R}^{u} \eta_{N_{S R}^{u}}^{k}
$$

In Eq. (38), $p_{S R}^{u}$ is defined by Eq. (13); $\eta_{N_{S R}^{u}}^{k}$ denotes the probability that the $k$ th relay is selected for receiving data at state $\mathbf{s}_{u}$, which has the similar form as $p_{N_{S R}^{u}}^{n}$. At the same time, the average queuing packet length at the relay $R_{k}$ is

$$
E\left\{\Psi\left(Q_{k}\right)\right\}=\sum_{u=1}^{(L+1)^{K}} \pi_{u} \Psi_{u}\left(Q_{k}\right)
$$

Hence, substituting Eqs. (38) and (39) into Eq. (37), we can obtain the average packet delay at relay $R_{k}$.

The above discussion indicates that the i.ni.d fading causes not only the degradation in diversity and coding gains but also the different average packet delays at relays. To achieve a perspective, in Tables 2 and 3, by taking $\omega_{S R_{k}}=\omega_{S R}, \omega_{R_{k} D}=\omega_{R D}$, and $\omega_{S R}=4-\omega_{R D}, k=1, \ldots$, $K$, we present the average packet delay comparison analysis. From Table 2, it is easy to see that when the conditions $\omega_{S R_{k}}=\omega_{S R}$ and $\omega_{R_{k} D}=\omega_{R D}$ are satisfied, the average data packet delays at all relay are same. This is due to the fact that under the identical distribution, each relay is selected with equal probability for receipt or transmission data. It is also seen that for the different values of $\omega_{S R} / \omega_{R D}$, the average packet delays at each relay are different greatly. We see that the average data packet delay is increasing with the value of $\omega_{S R} / \omega_{R D}$. When $\left(\omega_{S R} / \omega_{R D}\right)=1$, i.e., $\omega_{S R}=\omega_{R D}$, the average packet delays at all relays equal to $K L$. When $\left(\omega_{S R} / \omega_{R D}\right)>1$, the average packet delays are greater than $K L$. However, when $\left(\omega_{S R} / \omega_{R D}\right)<1$, the average packet delays are smaller than $K L$. This is not surprising because the higher relay-destination link gains imply that the relay-destination is more likely to be selected and the data packet are more quickly forwarded to the destination.

By taking $K=3, L=3$, and $m=2$, in Table 3 , we present the average packet delays at all relays under different channel realizations. These different $S-R$ link powers are arbitrarily generated by Gaussian random vector with mean $\mu=1.5$ and variance $\sigma^{2}=0.1,0.2,0.33,0.5$, 0.6, 0.8 and 0.9, respectively. Different from the results in Table 2, from Table 3, we see that the average packet delays at different relays are different entirely when the relay channels follow the non-identical distribution. Besides this, Table 3 also displays that, due to the different link powers for each system realization, the packet delays at different relays are also different greatly. For comparison analysis, in Table 3, we also present the total average delay defined by $\bar{D}_{R}=\frac{1}{K} \sum_{k=1}^{K} D_{R_{k}}$. Table 3 shows clearly that when $\sigma^{2} \leq 0.8$, the total average delay $\bar{D}_{R}$ is increasing with the disparity among the all $S-R$ link

\begin{tabular}{|c|c|c|c|c|c|c|c|c|c|}
\hline \multirow[t]{3}{*}{$\overline{\omega_{S R}}$} & \multirow[t]{3}{*}{$\omega_{R D}$} & \multicolumn{4}{|l|}{$P=0 d B$} & \multicolumn{4}{|l|}{$P=8 d B$} \\
\hline & & \multicolumn{2}{|l|}{$m=2$} & \multicolumn{2}{|l|}{$m=3$} & \multicolumn{2}{|l|}{$\overline{m=2}$} & \multicolumn{2}{|l|}{$m=3$} \\
\hline & & $\overline{D_{R 1}}$ & $D_{R 2}$ & $\overline{D_{R 1}}$ & $D_{R 2}$ & $\overline{D_{R 1}}$ & $D_{R 2}$ & $\overline{D_{R 1}}$ & $D_{R 2}$ \\
\hline 1 & 3 & 0.1552 & 0.1552 & 0.0449 & 0.0449 & 1.5854 & 1.5854 & 1.3705 & 1.3705 \\
\hline 1.5 & 2.5 & 1.3876 & 1.3876 & 0.9404 & 0.9404 & 2.5020 & 2.5020 & 2.2423 & 2.2423 \\
\hline 1.9 & 2.1 & 3.3951 & 3.3951 & 3.2379 & 3.2379 & 3.6681 & 3.6681 & 3.5945 & 3.5945 \\
\hline 1.99 & 2.01 & 3.9375 & 3.9375 & 3.9205 & 3.9205 & 3.9667 & 3.9667 & 3.9592 & 3.9592 \\
\hline 2 & 2 & 4 & 4 & 4 & 4 & 4 & 4 & 4 & 4 \\
\hline 2.01 & 1.99 & 4.0630 & 4.0630 & 4.0803 & 4.0803 & 4.0333 & 4.0333 & 4.0408 & 4.0408 \\
\hline 2.1 & 1.9 & 4.6545 & 4.6545 & 4.8461 & 4.8461 & 4.3322 & 4.3322 & 4.4056 & 4.4056 \\
\hline 2.5 & 1.5 & 8.6216 & 8.6216 & 11.1097 & 11.1097 & 5.4980 & 5.4980 & 5.7577 & 5.7577 \\
\hline 3 & 1 & 42.2118 & 42.2118 & 122.0643 & 122.0643 & 6.4148 & 6.4148 & 6.6296 & 6.6295 \\
\hline
\end{tabular}
powers.

Table 2 Average data packet delay (slots) $(K=2, L=2)$ 
Table 3 Average data delay (slots) $(K=3, L=3, m=2)$

\begin{tabular}{|c|c|c|c|c|c|c|c|c|c|}
\hline \multirow[t]{2}{*}{$\overline{\sigma^{2}}$} & \multirow[t]{2}{*}{$\omega_{S R}$} & \multicolumn{4}{|l|}{$P=O d B$} & \multicolumn{4}{|l|}{$P=8 d B$} \\
\hline & & $\overline{D_{R 1}}$ & $D_{R 2}$ & $D_{R 3}$ & $\bar{D}_{R}$ & $\overline{D_{R 1}}$ & $D_{R 2}$ & $D_{R 3}$ & $\bar{D}_{R}$ \\
\hline 0.1 & $1.3612,0.9315,1.7658$ & 1.8535 & 1.3082 & 2.8242 & 1.9953 & 3.0862 & 2.4753 & 3.9014 & 3.1543 \\
\hline 0.2 & $1.6428,1.6399,1.1132$ & 2.9223 & 2.9136 & 1.7735 & 2.5365 & 4.0000 & 3.9929 & 2.8869 & 3.6266 \\
\hline 0.33 & $1.7804,1.3981,1.3874$ & 3.6287 & 2.4657 & 2.4405 & 2.8456 & 4.5572 & 3.5689 & 3.5440 & 3.8900 \\
\hline 0.5 & $2.5036,1.7062,1.6399$ & 9.2559 & 6.7472 & 6.3987 & 7.4673 & 9.8060 & 7.5676 & 7.3297 & 7.9944 \\
\hline 0.6 & $1.7259,1.6532,2.7298$ & 7.5342 & 7.1323 & 9.5558 & 8.0741 & 8.5251 & 8.2828 & 9.4977 & 8.7685 \\
\hline 0.8 & $2.9201,0.7805,2.1231$ & 10.1753 & 5.5529 & 10.8074 & 8.8452 & 8.8741 & 8.7285 & 9.3704 & 8.9910 \\
\hline 0.9 & $2.2837,2.9486,1.9430$ & 15.0157 & 12.6763 & 14.2159 & 13.9693 & 13.4722 & 11.6383 & 13.5149 & 12.8751 \\
\hline
\end{tabular}

\section{Weighted-based MLS schemes}

The non-identical fading imposes the great impact on diversity and coding gains as well as the average packet delay. As a result, the MLS schemes may be unstable. Here, we consider a modified buffer-aided MLS scheme that can overcome the performance loss and degrade the packet delays at all relays over the i.ni.d fading channels. One of the ways of doing it is introducing two additional weight vectors $\boldsymbol{\omega}_{S R}^{w}$ and $\boldsymbol{\omega}_{R D}^{w}$, such that the selection of the best link in MLS schemes is based on the equivalent $S$ - $R$ link power vector $\boldsymbol{\omega}_{S R}^{E}=\boldsymbol{\omega}_{S R}^{w} \cdot \boldsymbol{\omega}_{S R}$ and the equivalent $R-D$ link power vector $\boldsymbol{\omega}_{R D}^{E}=\boldsymbol{\omega}_{R D}^{w} \cdot \boldsymbol{\omega}_{R D}$. In general, there are three methods to determine the weight vectors $\boldsymbol{\omega}_{S R}^{w}$ and $\boldsymbol{\omega}_{R D}^{w}$, which are referred as max-weight, min-weight, and mean-weight methods, respectively. Nevertheless, the weight vectors $\boldsymbol{\omega}_{S R}^{w}$ and $\boldsymbol{\omega}_{R D}^{w}$ can be given by the following form.

$$
\begin{aligned}
\boldsymbol{\omega}_{S R}^{w} & =\left\{\frac{1}{\omega_{S R_{1}}}, \ldots, \frac{1}{\omega_{S R_{K}}}\right\} \times W, \boldsymbol{\omega}_{R D}^{w} \\
& =\left\{\frac{\lambda}{\omega_{R_{1} D}}, \ldots, \frac{\lambda}{\omega_{R_{K} D}}\right\} \times W
\end{aligned}
$$

where $\lambda$ is a power adjusting factor so that the equivalent transmission power of $R-D$ links is greater than the one of $S-R$ links a litter. In practical implementation, the power adjusting factor $\lambda$ can be determined by the tolerable maximum delay and maximum outage probability. As a result, the delay performance of MLS schemes is improved greatly. For max-weight and min-weight schemes, in Eq. (40), we have $W=\max \left\{\boldsymbol{\omega}_{S R}, \boldsymbol{\omega}_{R D}\right\}$ and $W=\min \left\{\boldsymbol{\omega}_{S R}, \boldsymbol{\omega}_{R D}\right\}$, respectively. The max-weight and min-weight schemes are suitable for the scenario where the disparity of all relay channel powers (or variances) is small relatively. However, in practical implementation, due to the different relay positions and heavy fading, the disparity can be very large. To overcome this problem, we propose the mean-weight scheme, in which the weight factor $W$ is given by the following algorithm in Table 4.
The proposed algorithm can be implemented in a centralized or distributed manner. Here, we employ a hybrid distributed-centralized manner to select the best link. This is due to the fact that determining weight fact $W$ requires the statistical channel state information (CSI) of all links. At the same time, in practical communication systems, the distributed manner is preferable for the selection of the best link, especially in the case where the number of contention links is very large. For such hybrid manner, before each time slot, each relay transmits the statistical CSI to the central controller, which can be obtained by listening pilot signals from source and destination. After receiving the statistical CSI from all relays, the central controller performs the algorithm given in Table 4 and broadcasts the result to all relays. Upon receiving the signal from central controller, each relay then starts a timer by jointly considering the buffer sates and its input $(S-R)$ and output $(R-D)$ link strength as well as the weight factor $W$ obtained from central controller. The duration of timer is a proportional function that depends on the weight factor $W$ and $S-R$ or $R-D$ link strength. As a result, the best link can be selected.

Note that, due to the introduction of weight factor $W$, the selection of the best link in W-MLS schemes is based on the equivalent $S-R$ link power vector $\boldsymbol{\omega}_{S R}^{E}=\boldsymbol{\omega}_{S R}^{W} \cdot \boldsymbol{\omega}_{S R}$ and the equivalent $R-D$ link power vector $\boldsymbol{\omega}_{R D}^{E}=\boldsymbol{\omega}_{R D}^{w} \cdot \boldsymbol{\omega}_{R D}$. Therefore, the W-MLS obtains the following advantages over the conventional MLS schemes [26]. Firstly, all $S-R$ and $R-D$ links are selected with equal probability so that the maximum diversity and coding gains can be achieved. Secondly, at all relays, the average packet delays are same. Therefore, our W-MLS schemes overcome the unbalance caused by the no-identical fading. Finally, in our W-MLS schemes, the average packet delays can be further decreased by adjusting $\lambda$ as shown in Table 4 .

Of cause, the superiority of W-MLS over the conventional MLS is achieved at the price of system complexity. This is because that the weigh factor $W$ must be 
Table 4 Algorithm determining the weight factor $W$ in mean-weight scheme

Require: The channel variance vectors $X=\left\{\boldsymbol{\omega}_{S R}, \boldsymbol{\omega}_{R D}\right\}$, the threshold $\mu$, and the power

adjusting factor $\lambda . \% X=\left\{\boldsymbol{\omega}_{S R}, \boldsymbol{\omega}_{R D}\right\}$ denoting a new vector that contain all

$\%$ elements in vectors $\boldsymbol{\omega}_{S R}$ and $\boldsymbol{\omega}_{R D}$

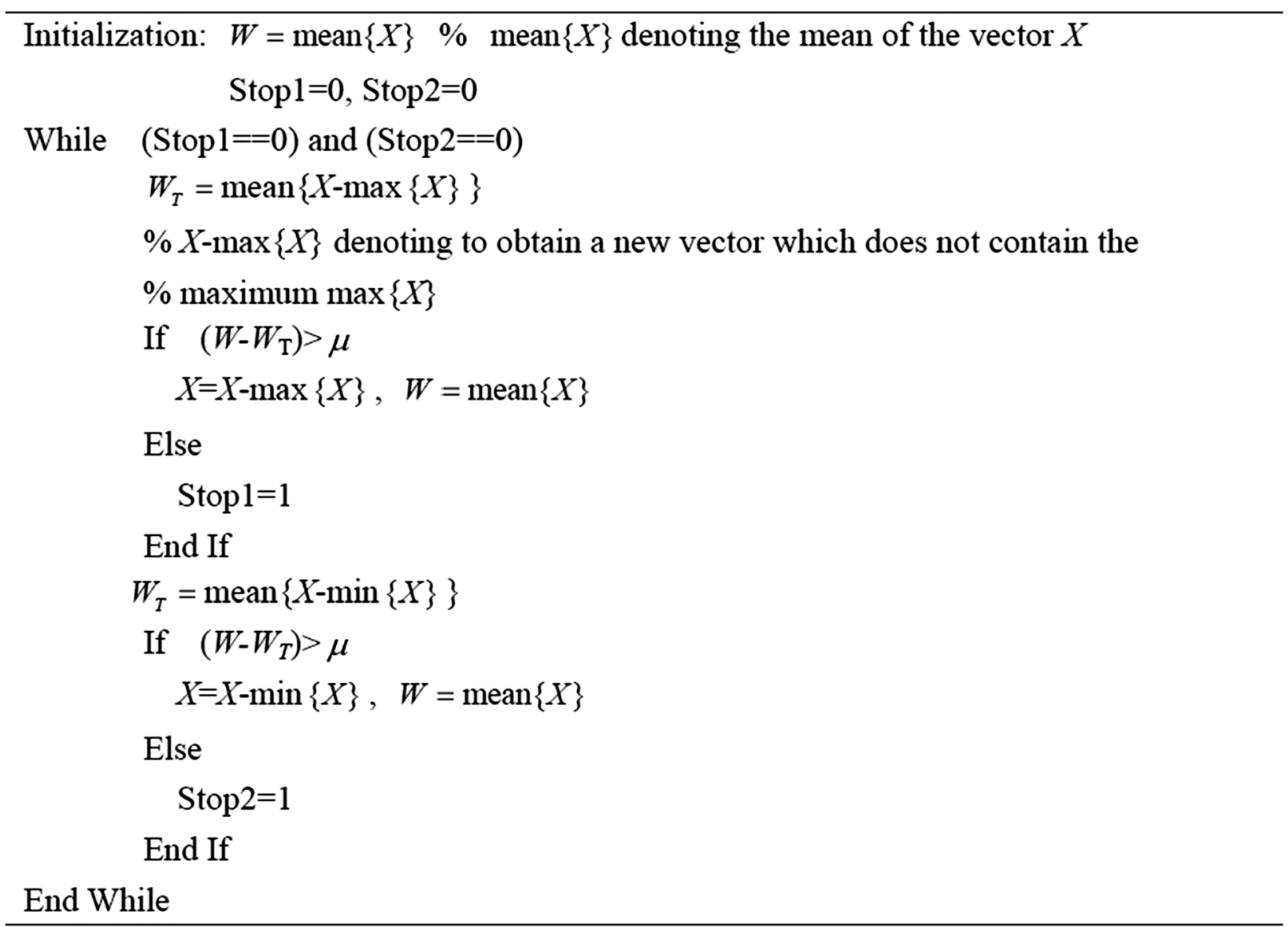

Output: $W$

determined firstly before performing the best link selection. From Table 4, we see that when the mean-weight MLS scheme is employed, the complexity depends on the disparity of channel gains. That is to say, the complexity is increasing with the disparity. On the contrary, as a candidate of complexity-performance trade-off, the max-weight or min-weight scheme has low employment complexity. Obviously, when the disparity of channel gains is high, the mean-weight MLS should be performed so that the system performance is improved greatly. When the disparity of channel gains is small, the max-weight or min-weight scheme is a good selection with low implementation complexity and improved performance.

\section{Numerical results and simulations}

Based on the previous analysis and derivations, the simulated and numerical results are presented in this section. To obtain a comprehensive comparison, the T-BRS and MMLS schemes are also analyzed in this section. For simplicity, throughout the simulations, we take the system spectrum efficiency $R=1 \mathrm{bits} / \mathrm{s} / \mathrm{Hz}$. We also 
assume that the fading severity factors are same, i.e., $m_{S R_{k}}=m_{R_{k} D}=m$, for all Nakagami- $m$ fading links.

By taking the number of relays $K=2$ and the fading severity factor $m=2$, in Fig. 3, we first investigate the outage probability versus the transmit power $P$ with different size $L$ of relay buffers over the i.i.d Nakagami- $m$ fading channels. Specially, we take the buffer's size $L=2,5,10,20$, and 30, and $\left\{\omega_{S R_{k}}\right\}_{k=1}^{K}=\left\{\omega_{R_{k} D}\right\}_{k=1}^{K}=1$. From Fig. 3a, it is clearly shown that the MLS schemes always outperform the T-BRS and MMLS schemes when the relay channels follow the i.i.d Nakagami- $m$ fading. The MLS schemes provide not only the coding gain but also the diversity gain over the T-BRS and MMLS schemes. Moreover, the achievable diversity gain by MLS over TBRS and MMLS is increasing with the increase of the buffer's size $L$. To further illustrate the impact of the fading severity factor $m$ on the diversity order, in Fig. 3b, the outage probability is investigated by taking the fixed buffer size $L=10$ and taking the variable fading severity factor $m$, $m=3,4$. It is found that the performance of the three buffer-aided relaying schemes is enhanced by the increase of $m$. Similar to Fig. 3a, we see that for each fixed $m$ in Fig. 3b, the MLS schemes always outperform the T-BRS and MMLS ones. This is due to the superiority provided by the MLS schemes.

In Fig. 4, the outage probability of the three bufferaided relaying schemes is investigated over more general i.ni.d Nakagami- $m$ fading channels, in which there are two different channel realizations are considered, respectively. Different from the results achieved in Fig. 3, from Fig. 4, we achieve that when the relaying channels experience the non-identical fading, all curves in Fig. 4a have the same slope, approximately. This observation indicates that in this non-identically distributed fading case, the MLS scheme does not provide the diversity gain over the T-BRS and MMLS schemes, or only givens very limited diversity gain. At the same time, it is also achieved that although the MLS schemes outperform the T-BRS, it has the same performance as MMLS schemes in certain case. For example, Fig. $4 \mathrm{~b}$ shows that the achieved coding gains by MLS over MMLS are nominal. Obviously, the performance loss is increasing with the disparity of the channel variances. Therefore, we have the following results. (1) When all relaying channel experience i.i.d fading, compared with the both T-BRS and MMLS schemes, the MLS schemes provide not only the coding gain but also the diversity gain. Moreover, the achievable diversity order is increasing with the buffer's size $L$. (2) When all relaying channels experience i.ni.d fading, the performance of the MLS schemes is impaired greatly by the disparity of relaying channels. On the one hand, the MLS schemes only provide the very limited diversity gain over the T-BRS and MMLS schemes. In an extreme case, there is no diversity gain achieved even if the buffer size $L$ is large enough. On the other hand, the achieved coding gain is also limited. Specially, in certain case, the MLS schemes can be inferior to the MMLS schemes even if the MLS schemes always achieve the coding gain over the T-BRS.

In Fig. 5, we plot the average packet delay versus the transmit power $P$ under different $m$ for the case $\omega_{S R_{k}}=\omega_{S R}, \quad \omega_{R_{k} D}=\omega_{R D}, \omega_{S R} \neq \omega_{R D}, k=1, \ldots, K$. Comparing Fig. 5a, b, we find that, for the two considered cases, $\omega_{S R}<\omega_{R D}$ and $\omega_{S R}>\omega_{R D}$, the system parameters $P$ and $m$ impose different impact on the average packet delay. When $\omega_{S R}<\omega_{R D}$, the average packet delay is proportional inversely with the fading severity factor $m$. This observation indicates that when the condition $\omega_{S R}<\omega_{R D}$ is satisfied, the systems with larger $m$ obtain not only higher diversity gain but also

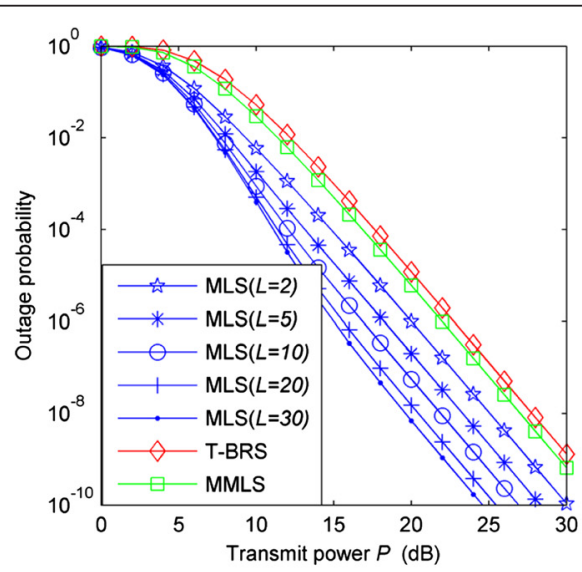

(a) With variable $L$ and fixed $m$

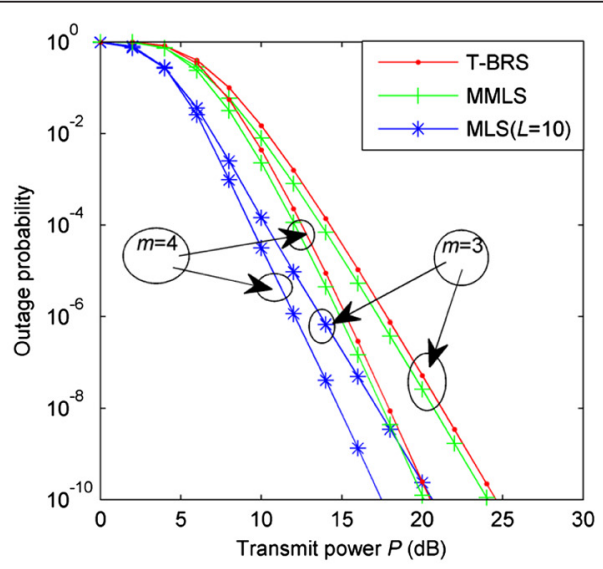

(b) With fixed $L$ and variable $m$

Fig. 3 Outage performance versus transmit power $P$ over i.i.d fading channels 

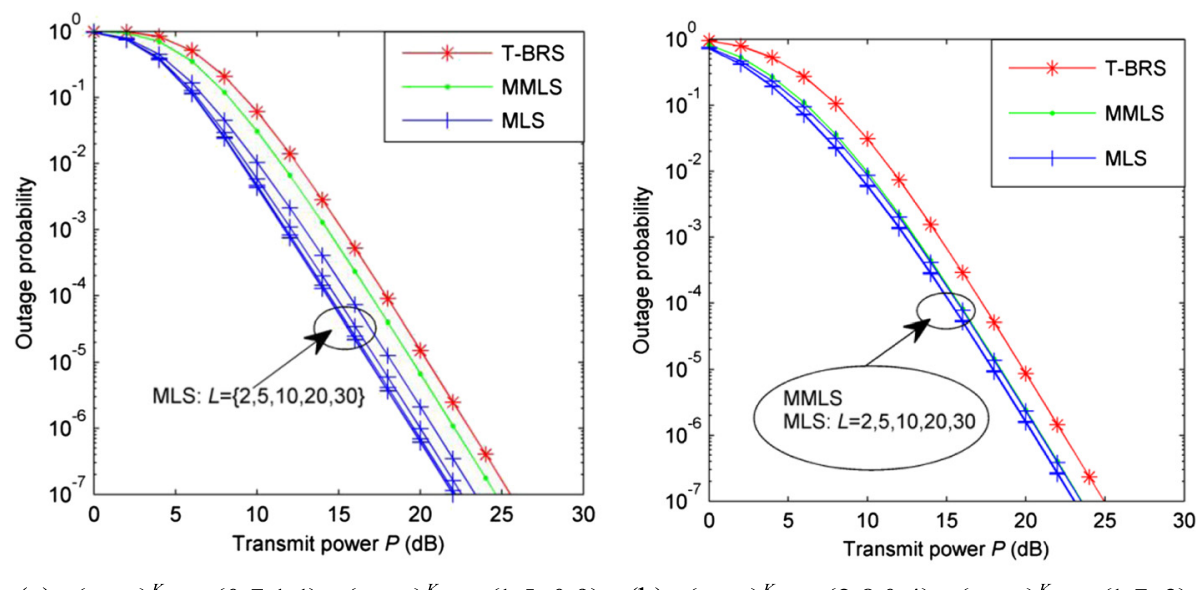

(a) $\left\{\omega_{S R_{k}}\right\}_{k=1}^{K}=\{0.7,1.1\},\left\{\omega_{R_{k} D}\right\}_{k=1}^{K}=\{1.5,0.9\}$

(b) $\left\{\omega_{S R_{k}}\right\}_{k=1}^{K}=\{2.8,0.4\},\left\{\omega_{R_{k} D}\right\}_{k=1}^{K}=\{1.7,3\}$

Fig. 4 Outage performance versus transmit power $P$ over i.ni.d fading channels $(m=2)$

less average packet delay. However, when $\omega_{S R}>\omega_{R D}$, compared with Fig. 5a, Fig. 5b shows that the average packet delay is increasing with $m$. For the effect of the transmit power $P$, Fig. $5 \mathrm{a}$, b shows that when the transmit power $P$ is large enough, the average packet delay is not affected by $P$ and saturated to a constant. The reason is that when the transmit power $P$ is large enough, the difference of equivalent SNRs between the $S-R$ and $R-D$ links is negligible, such that all links are selected with equal probability and the delay approaches a constant. On the contrary, when the transmit power $P$ is small relatively, Fig. 5 a shows that the increase of $P$ causes the increase of the average packet delay, while Fig. 5b shows that it causes the decrease of the average packet delay.
Similar to Fig. 5, to observe the average packet delays at different relays over non-identical fading channels, by taking $\left\{\omega_{S R_{K}}\right\}_{k=1}^{K}=\{1.3612,0.9315,1.7658\}$ and $\left\{\omega_{S R_{K}}\right\}_{k=1}^{K}=\{2.6388,3.0685,2.2342\}$, the corresponding average packet delays versus the transmit power are plotted in Fig. 6a, b, respectively. The figure shows clearly that when the relay channels obey nonidentical distribution, the delays at different relays are different. Moreover, the asymptotic delays are also different when the transmit power goes large enough.

To illustrate the feasibility and superiority of the proposed weight-based MLS schemes, in Figs. 7 and 8, we compare the outage performance and average packet delays among the weight-based and non-weight-based schemes, where we take $K=2, m=2, \boldsymbol{\omega}_{S R}=\{1,0.3\}$,

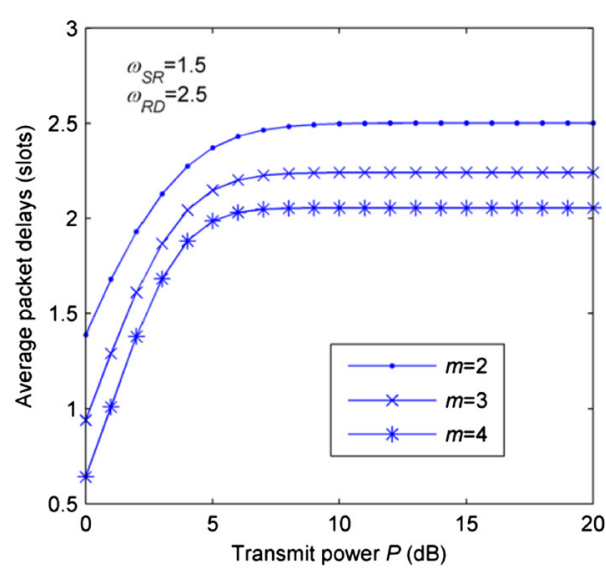

(a) $\omega_{S R}<\omega_{R D}$

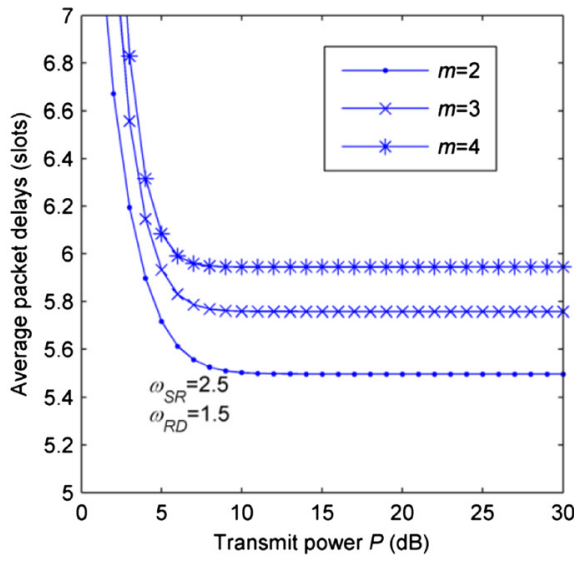

(b) $\omega_{S R}>\omega_{R D}$

Fig. 5 Average packet delays versus transmit power $(L=2, K=2)$ 


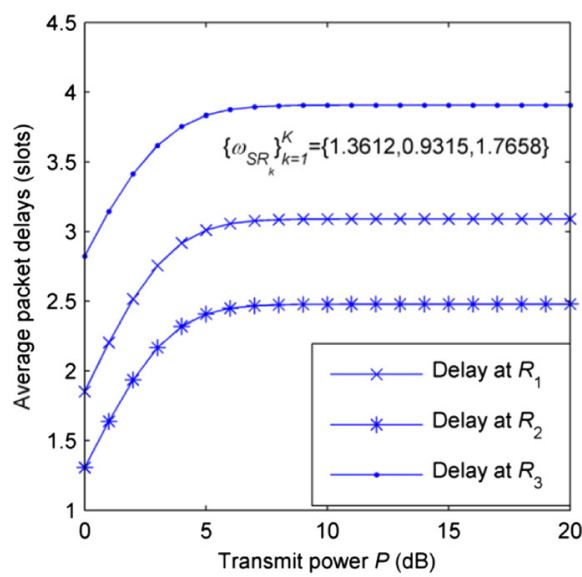

(a)

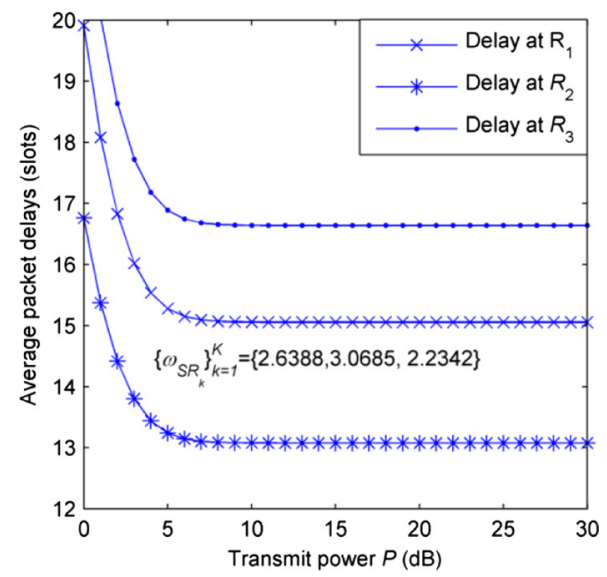

(b)

Fig. 6 Average packet delays versus transmit power at different relays $(K=3, L=3, m=2)$

$\boldsymbol{\omega}_{R D}=1-\boldsymbol{\omega}_{S R}$. In Figs. 7 and 8, "W" stands for the proposed weight-based schemes, while "NW" stands for the non-weight-based schemes. For a fair comparison, the following power constraint is adopted.

$$
\frac{P_{W}}{2 K}\left[\sum_{k=1}^{K} \frac{W}{\omega_{S R_{k}}}+\sum_{k=1}^{K} \frac{W \lambda}{\omega_{R_{k} D}}\right]=P
$$

where $P_{W}$ denotes that transmission power at each node when the proposed weight-based schemes is used to select the best link (or relay). In the two figures, we only consider the T-BRS and MLS schemes with or without the proposed weight-based method. From Fig. 7a, we see that the effect of weight-based schemes on the outage performance of both the T-BRS and MLS schemes is different greatly. Specially, for the T-BRS schemes, it is easily seen that the proposed weight-based schemes do not improve outage performance, instead of increasing the outage probability, especially in the low region of transmission power $P$. Only in medium and

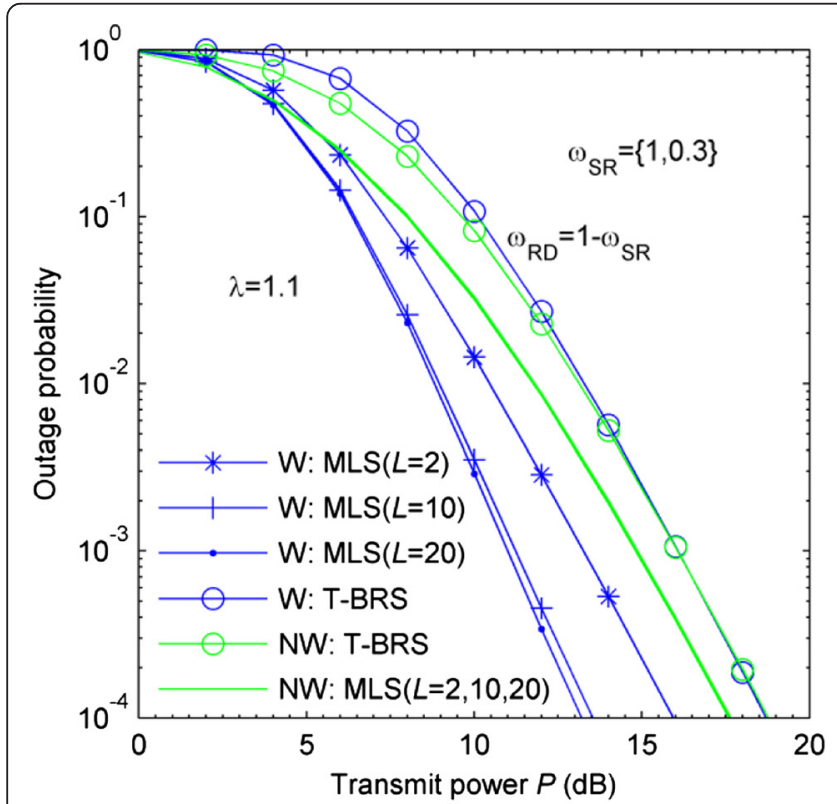

(a) Outage probability

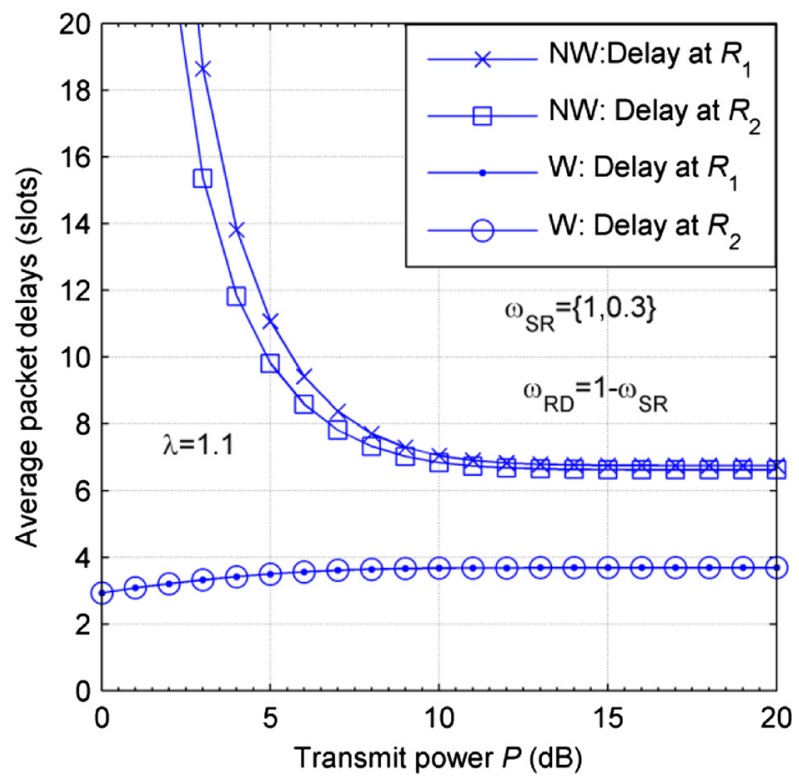

(b) Average packet delays $(L=2)$

Fig. 7 Comparison of outage and delay performance between the weight-based and non-weight-based schemes $(m=2, K=2, \lambda=1.1, N W$ nonweight-based schemes, $W$ weight-based schemes) 


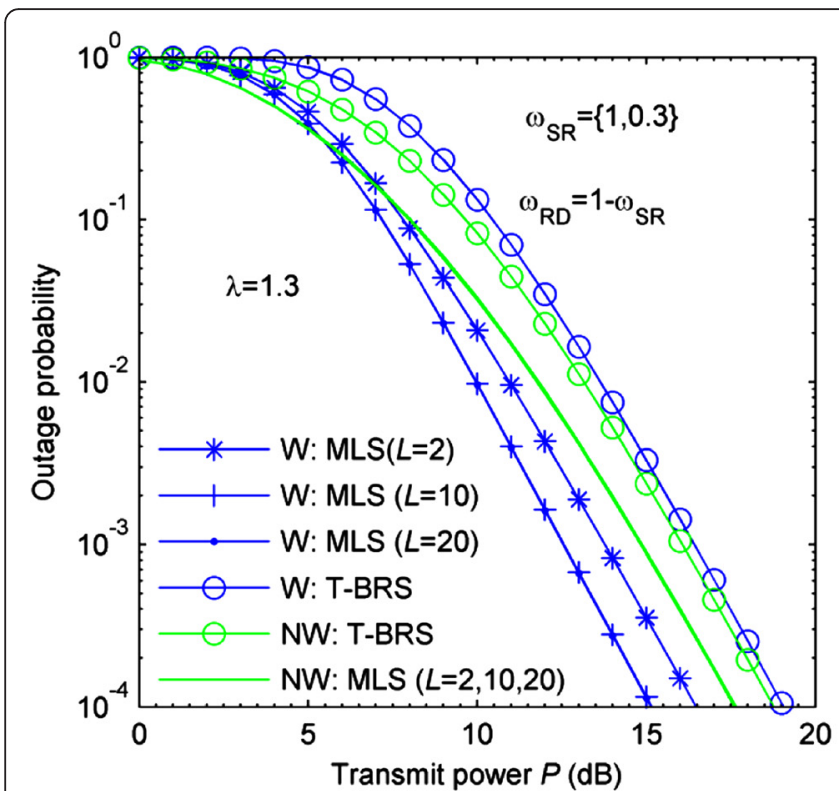

(a) Outage probability

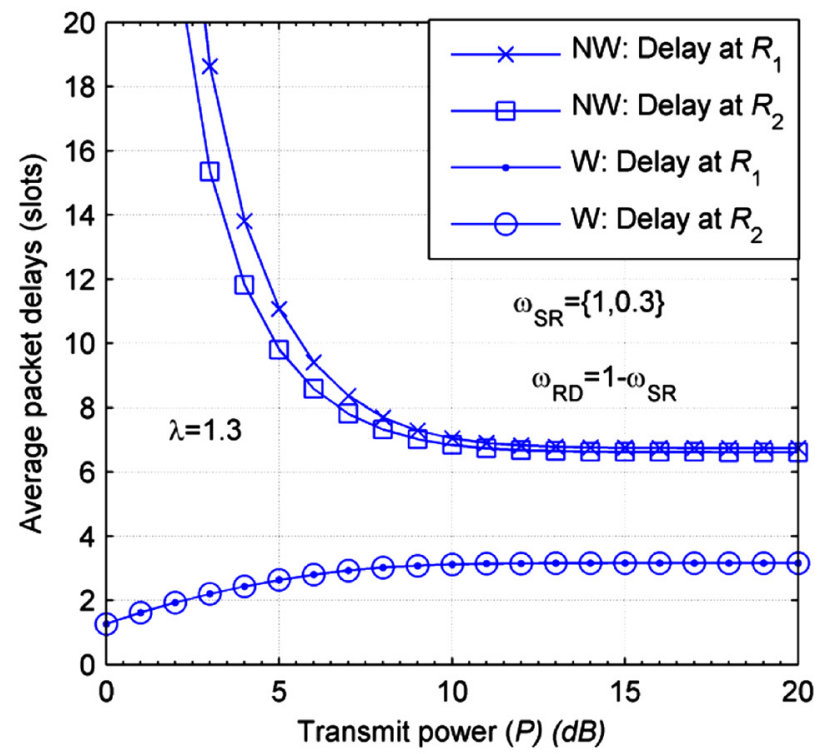

(b) Average packet delays $(L=2)$

Fig. 8 Comparison of outage and delay performance between the weight-based and non-weight-based schemes $(m=2, K=2, \lambda=1.3$, NW non-weight-based schemes, $W$ weight-based schemes)

high region of $P$, the loss will be reduced gradually. In high $P$, the weight-based schemes obtain the same outage performance as the non-weighted-based ones. However, for the MLS schemes, it is clearly found that the proposed weight-based methods cause greatly improvement on outage performance over the entire region of transmission power $P$. For example, Fig. 7 a shows that when the power adjusting factor $\lambda=1.1$ and $L=20$, the weight-based MLS schemes achieve the power gain of $5 \mathrm{~dB}$ over the traditional MLS schemes at $10^{-4}$ of outage probability. At the same time, another interesting result can also be found in Fig. 7b. Figure 7b shows that, with the proposed weight-based MLS, the packet delays at all relays are improved greatly. On the one hand, the delays at all relays are the same entirely, which indicates that all data packets can be forwarded to destination with equal probability. On the other hand, the average packet delay at each relay is reduced distinctly, especially in low region of transmit power $P$. Therefore, it is obtained that the proposed weight-based schemes not only can improve the outage performance but also can decrease the average packet delay. The proposed W-MLS schemes are feasible.

For more insight, in Fig. 8, the outage and delay performance are compared once again. In the figure, we take the power adjusting factor $\lambda=1.3$. Comparing Figs. $7 \mathrm{a}$ and $8 \mathrm{a}$, we see that the increase of the power adjusting factor $\lambda$ yields the loss in the outage performance of the weight-based MLS schemes. For example, when $L=20$, Fig. 8a shows that the weight-based MLS schemes only achieve the power gain of $3 \mathrm{~dB}$ over the traditional MLS schemes at $10^{-4}$ of outage probability. On the contrary, Fig. $8 \mathrm{~b}$ shows that the average packet delays for the proposed weight-based MLS schemes are decreasing with the power adjusting factor $\lambda$. This is due to the fact that the outage probability of relay systems is increasing with the symmetricity of relay channels. When the channels are symmetric entirely, the relay systems achieve the optimal outage performance. Therefore, we have the result that the increasing power factor yields the increasing outage probability. However, when the equivalent power of $R-D$ links is greater than the one of $S-R$ links, the probability that one $R-D$ link is selected is greater than the one that one $S-R$ link is did. The results from Figs. 7 and 8 indicate that in practical application, the power adjusting factor $\lambda$ should be jointly determined by the maximum delay, maximum outage probability, etc. As a result, the proposed W-MLS scheme is a delay-diversity trade-off scheme.

\section{Conclusions}

While it has been proved that in buffer-aided relaying systems, the MLS schemes can provide both the diversity and coding gains over the T-BRS and MMLS schemes when the all relay channels obey the i.i.d fading, the corresponding diversity and delay performance of the MLS schemes have not been reported when the relay channels experience the practical i.ni.d fading. Thus, in 
this paper, the MLS schemes are investigated over the i.ni.d Nakgami- $m$ channels. By employing Markov chain model, the statistical descriptions of the MLS schemes are achieved firstly. Then, with the derived statistical descriptions, the average packet delays are investigated over the i.ni.d Nakgami- $m$ fading channels. The obtained results show that the non-identically distributed relay links impose a severe loss in the diversity and coding gains for the MLS schemes. Moreover, when the disparity of the powers of relay links is very large, the MLS schemes no longer provide any diversity gain over the TBRS and MMLS ones even if the buffers' size is enough large. However, when the disparity of the powers of relay links is little relatively, the enough diversity and coding gains can be still provided by the MLS schemes. For the average packet delays, it is achieved that under the i.ni.d fading channels, the average packet delays at all relays are different. The results indicate that at some relays, the average packet delays can be very large, and at other relays, they are small. This phenomenon yields that at some relays, the data packets would be trapped for a long time and the relay systems with MLS schemes are unstable. However, it is enough lucky that when the powers of $S-R$ links are less than the ones of $R-D$ links, the average packet delays are reduced evidently. Inspired this observation, a W-MLS diversity-delay trade-off scheme is proposed to reduce the delay and to enhance the stability of MLS schemes but still obtain the enough diversity and coding gains over T-BRS and MMLS schemes. In the proposed W-MLS schemes, before selecting a best link, the systems multiply the link gains by a weight vector, such that the equivalent signal-to-noise ratios of $S-R$ links are same, so does the ones of $R-D$ links, such that the data packet delays at all relays are same. By adjusting the weight vector, it is guaranteed that the equivalent SNRs of $R-D$ links are greater than the ones of $S-R$ links, such that the delays can be decreased evidently. Moreover, by designing feasible weight vector, the W-MLS can still provide the enough diversity and coding gains.

\section{Appendix 1: Proof of Eq. (13)}

With the definition $\gamma_{S R}^{u}=\underbrace{\max }_{k \in N_{S R}^{u}}\left\{\gamma_{S R_{k}}\right\}$ and $\gamma_{S R_{k}}$ following the Nakagami- $m$ distribution with parameters $m_{S R_{k}}$ and $\omega_{S R_{k}}$, in Eq. (12) the term $\operatorname{Pr}\left\{\gamma_{S R}^{u}<y\right\}$ is given by

$$
\operatorname{Pr}\left\{\gamma_{S R}^{u} \leq y\right\}=\prod_{k=1}^{\left|N_{S R}^{u}\right|}\left(1-\sum_{i=0}^{m_{S R_{N R}}^{u}(k)} \frac{1}{i !}\left(\frac{m_{N_{S R}^{u}}^{u}(k)}{\omega_{N_{S R}^{u}(k)}}\right)^{i} e^{i-\frac{m_{S R}^{u}(k)}{\omega_{N}^{u}} S_{S R}^{u}(k)}\right)
$$

where $N_{S R}^{m}(k)$ denotes the $k$ th element of the set $N_{S R}^{m}$. For example, if $N_{S R}^{m}=\{1,3,5,6\}$, then we have $N_{S R}^{m}$
$(2)=3$. Furthermore, according to [35] the Eq. (42) can been written as

$$
\begin{aligned}
& \operatorname{Pr}\left\{\gamma_{S R}^{u} \leq y\right\}=\sum_{k=0}^{\left|N_{S R}^{u}\right|} \frac{(-1)^{k}}{k !} \underbrace{\sum_{q_{1}=1}^{N_{S R}^{u} \mid} \cdots \sum_{q_{k}=1}^{N_{S R}^{u} \mid}}_{q_{1} \neq \ldots \neq q_{k}} \underbrace{\sum_{h_{1}+\cdots+h_{k}=j}^{k}\left(m_{N_{S R}^{u}\left(q_{l}\right)^{-1}} \sum_{j=0}\right.}_{l=1} \\
& \prod_{t=1}^{k} \frac{1}{\left(h_{t}\right) !}\left(\frac{m_{N_{S R}^{u}\left(q_{t}\right)}}{\omega_{N_{S R}^{u}\left(q_{t}\right)}}\right)^{h_{t}} y^{k} y^{t=1} e^{-\sum_{t=1}^{k} \frac{m_{N_{S R}^{u}\left(q_{t}\right)}}{\omega_{N_{S R}^{u}\left(q_{t}\right)}^{u}} y}
\end{aligned}
$$

At the same time, to obtain the closed-form expression of $f_{\gamma_{R D}^{u}}(y)$ in Eq. (12), the CDF $F_{\gamma_{R D}^{u}}(y)$ of $\mathrm{RV} \gamma_{R D}^{u}$ is required. To this end, we first consider its $\operatorname{CDF} F_{\gamma_{R D}^{u}}(y)$. With $\gamma_{R_{k} D}$ following Nakagami- $m$ distribution with parameters $m_{R_{k} D}$ and $\omega_{R_{k} D}$, having

$$
\begin{aligned}
& F_{\gamma_{R D}^{u}}(y)=\operatorname{Pr}\left\{\gamma_{R D}^{u} \leq y\right\}
\end{aligned}
$$

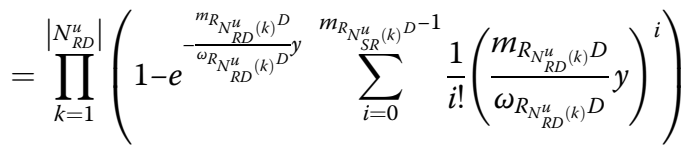

Taking the derivative of $F_{\gamma_{R D}^{u}}(y)$ with respect to $y$, we have the $\operatorname{PDF} f_{\gamma_{R D}^{u}}(y)$ given by

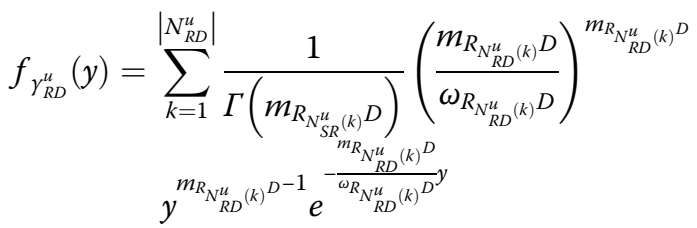

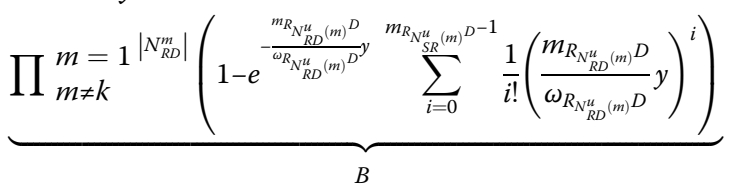

Similar to Eq. (43), using multinomial expansion leads to the term $B$ in Eq. (45) is given by

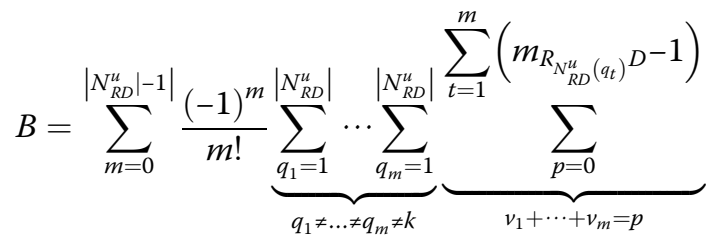

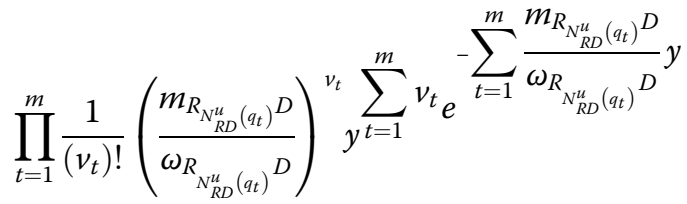


Thus, by substituting Eqs. (46), (45, and (43) into Eq. (12) and using (3.351.3) in [34], we have the probability $p_{S R}^{m}$ given by Eq. (13).

\section{Appendix 2: Proof of Eq. (17)}

According to Eq. (16), at state $\mathbf{s}_{u}$ the probability $p_{N_{S R}^{u}}^{n}$ is formulated as

$$
p_{N_{S R}^{u}}^{n}=\int_{0}^{\infty} \operatorname{Pr}\left\{Y_{N_{S R}^{u}(a)} \leq y\right\} f_{\gamma_{N_{S R}^{u}(a)}}(y) d y
$$

In Eq. (47), $f_{\gamma_{N_{S R}^{m}}(a)}($.$) is the PDF of \gamma_{N_{S R}^{u}(a)}$. With the definition $Y_{N_{S R}^{u}(a)}=\max _{k \in N_{S R}^{u}, k \neq a}\left\{\gamma_{S R_{N_{S R}^{u}(k)}}\right\}$, the $\operatorname{CDF} F_{Y_{N_{S R}^{u}(a)}}$ $(y)$ of $Y_{N_{S R}^{u}(a)}$ is

$$
\begin{aligned}
F_{Y_{S R}^{u}(a)}(y)=\left.\prod_{i \neq a}^{i=1}\right|^{\left|N_{S R}^{u}\right|} & \left(1-\frac{1}{\Gamma\left(m_{S R_{N_{S R}^{u}}^{u}(i)}\right)}\right. \\
& \left.\times \Gamma\left(m_{S R_{N_{S R}^{u}}^{u}(i)}, \frac{m_{S R_{N_{S R}^{u}(i)}}}{\omega_{S R_{N_{S R}^{u}}(i)}} y\right)\right)
\end{aligned}
$$

Using the results in [35] and [36] we have

$$
\begin{aligned}
& F_{Y_{N_{S R}(a)}(a)}(y)=\sum_{i=0}^{\left|N_{S R}^{u}\right|-1} \frac{(-1)^{i}}{i !} \underbrace{\sum_{f_{1}=1}^{N_{S R}^{u} \mid} \ldots \sum_{f_{i}=1}^{\mid N_{S R}^{u}}}_{f_{1} \neq \ldots \neq f_{i} \neq a} \underbrace{\sum_{t=1}^{i}\left(m_{S R N_{S R}^{m}\left(f_{t}\right)}-1\right)}_{g_{1}+\cdots+g_{k}=j} \\
& \prod_{t=1}^{i} \frac{1}{\left(g_{t}\right) !}\left(\frac{m_{S R N_{S R}^{u}\left(f_{t}\right)}}{\omega_{S R N_{S R}^{u}\left(f_{t}\right)}}\right)^{g_{t}} \sum_{t=1}^{i} g_{t} e^{-\sum_{t=1}^{i} \frac{m_{S R N_{S R}^{m}\left(f_{t}\right)}}{\omega_{S R N_{S R}^{m}\left(f_{t}\right)}}}
\end{aligned}
$$

At the same time, the PDF of the RV $\gamma_{N_{S R}^{u}(a)}$ is given by

$$
\begin{aligned}
& f_{\gamma_{S R_{N R}^{u}}^{u}(a)}(y)=\frac{1}{\Gamma\left(m_{S R_{N_{S R}^{u}}^{u}(a)}\right)}\left(\frac{m_{S R_{N_{S R}^{u}}^{u}(a)}}{\omega_{S R_{N_{S R}^{u}}^{u}(a)}}\right)^{m_{S R_{N} N_{S R}^{u}(a)}} \\
& \times y^{m_{S R_{N R}}^{u}(a)^{-1}} e^{-{ }^{m_{S R_{N} N_{S R}^{u}}(a)}{ }^{-\omega_{S R_{N R}}^{u}(a)}} y
\end{aligned}
$$

Substituting Eqs. (50) and (49) into (47), after some algebraic manipulations, we have the closed-form expression of $p_{N_{S R}^{m}}^{n}$ that is given by Eq. (17).

\section{Competing interests}

The authors declare that they have no competing interests.

\section{Acknowledgements}

The authors thank the editors and the anonymous reviewers for their constructive comments and suggestions, which helped to improve the quality of this paper. This work was supported by the Natural Science Foundation of China under Grant 61261015, the 973 project 2013CB329104, the Natural Science Foundation of China under Grant 61561043, 61372124, and 61302100, the Natural Science Foundation of Gansu Province for Distinguished Young Scholars (1308RJDA007), and the Foundation Research Funds for the University of Gansu Province: "Massive MIMO channels modelling and estimation over millimeter wave band for 5G".

\section{Author details}

${ }^{1}$ College of Computer Science and Engineering, Northwest Normal University, Lanzhou 730070, China. 'Wireless Communication Key Lab of Jiangsu Province, Nanjing University of Posts and Telecommunications, Nanjing 210003, China. ${ }^{3}$ Key Lab of Broadband Wireless Communication and Sensor Network Technique of Ministry of Education, Nanjing University of Posts and Telecommunications, Nanjing 210003, China.

Received: 11 September 2015 Accepted: 21 December 2015 Published online: 27 January 2016

\section{References}

1. J Xiangdong, Y Longxiang, F Haiyang, Tight performance bounds for twoway opportunistic amplify-and-forward wireless relaying networks with TDBC protocols. EURASIP J. Wirel. Commun. Netw. 2011, 1-8 (2011)

2. J Xiangdong, Y Longxiang, Upper and lower bounds of two-way opportunistic amplify-and-forward relaying channels. IEEE Commun Lett 16(8), 1180-1183 (2012)

3. ECVD Meulen, Three-terminal communication channels. Adv. Appl. Probab. 3(1), 120-154 (1971)

4. T Cover, AE Gamal, Capacity theorems for the relay channel. IEEE Trans. Inf. Theory IT-25(5), 572-584 (1979)

5. A Host-Madsen, Z Junshan, Capacity bounds and power allocation for wireless relay channels. IEEE Trans. Inf. Theory 51(6), 2020-2040 (2005)

6. A Sendonaris, E Erkip, B Aazhang, User cooperation diversity_-Part I. System description. IEEE Trans. Commun. 51(11), 1927-1938 (2003)

7. A Sendonaris, E Erkip, B Aazhang, User cooperation diversity—Part II. Implementation aspects and performance analysis. IEEE Trans. Commun. 51(11), 1939-1948 (2003)

8. J Laneman, D Tse, G Wornell, Cooperative diversity in wireless networks: efficient protocols and outage behavior. IEEE Trans. Inf. Theory 50(12), 3062-3080 (2004)

9. A Bletsas, A Khisti, DP Reed, A Lippman, A simple cooperative diversity method based on network path selection. IEEE J. Sel. Areas Commun. 24(3), 659-672 (2006)

10. JN Laneman, GW Wornell, Distributed space-time-coded protocols for exploiting cooperative diversity in wireless networks. IEEE Trans. Inf. Theory 49(10), 2415-2425 (2003)

11. CXu, S Ting-wai, QF Zhou, FCM Lau, High-SNR analysis of opportunistic relaying based on the maximum harmonic mean selection criterion. IEEE Signal Process Lett. 17(8), 719-722 (2010)

12. I Krikidis, J Thompson, S McLaughlin, N Goertz, Max-min relay selection for legacy amplify-and-forward systems with interference. IEEE Trans. Wirel. Commun. 8(6), 3016-3027 (2009)

13. L Yingbin, W Veeravalli, HV Poor, Resource allocation for wireless fading relay channels: max-min solution. IEEE Trans. Inf. Theory 53(10), 3432-3453 (2007)

14. X Bing, F Yijia, J Thompson, HV Poor, Buffering in a three-node relay network. IEEE Trans. Wirel. Commun. 7(11), 4492-4496 (2008)

15. N Zlatanov, R Schober, P Popovski, Throughput and diversity gain of bufferaided relaying, in PROC Of 2011 IEEE Global Communications Conference, 2011, pp. 1-6

16. N Zlatanov, R Schober, Buffer-aided half-duplex relaying can outperform ideal full-duplex relaying. IEEE Commun. Lett. 17(3), 479-482 (2013)

17. A El Shafie, MG Khafagy, A Sultan, Optimization of a relay-assisted link with buffer state information at the source. IEEE Commun. Lett. 18(12), 2149-2152 (2014)

18. S Wei, Y Jian, Y Qinghai, K Kyungsup, Auction based energy efficient link selection for buffer-aided relay networks, in PROC Of Communications and Information Technologies (ISCIT), 2014 14th International Symposium on, 2014, pp. 218-222 
19. N Zlatanov, A Ikhlef, T Islam, R Schober, Buffer-aided cooperative communications: opportunities and challenges. IEEE Commun. Mag. 52(4), 146-153 (2014)

20. T Islam, DS Michalopoulos, R Schober, V Bhargava, Delay constrained bufferaided relaying with outdated CSI, in Proc of Wireless Communications and Networking Conference (WCNC), 2014 IEEE, 2014, pp. 875-880

21. T Islam, A Ikhlef, R Schober, VK Bhargava, Diversity and delay analysis of buffer-aided BICM-OFDM relaying. IEEE Trans. Wirel. Commun. 12(11), 5506-5519 (2013)

22. D Chen, Z Jing, Y Lie-Liang, H Yongkai, N SoonXin, L Hanzo, Energy-efficient buffer-aided relaying relying on non-linear channel probability space division, in Proc Of Wireless Communications and Networking Conference (WCNC), 2014 IEEE, 2014, pp. 1979-1984

23. L Guan-Xing, D Chen, L Dong, L Guangtao, Z Yonghui, Outage analysis of dual-hop transmission with buffer aided amplify-and-forward relay, in Proc Of Vehicular Technology Conference (VTC Fall), 2014 IEEE 80th, 2014, pp. 1-5

24. M Darabi, B Maham, Z Yan, Buffer-aided link selection for incremental relaying systems, in Proc. of Computers and Communication (ISCC), 2014 IEEE Symposium on, 2014, pp. 1-6

25. D Chen, Y Lie-Liang, Z Jing, N Soon Xin, L Hanzo, Energy, delay, and outage analysis of a buffer-aided three-node network relying on opportunistic routing. IEEE Trans. Commun. 63(3), 667-682 (2015)

26. A Ikhlef, DS Michalopoulos, R Schober, Buffers improve the performance of relay selection, in Proc. of 2011 Global Telecommunications Conference (GLOBECOM 2011), 2011 IEEE, 2011, pp. 1-6

27. A Ikhlef, DS Michalopoulos, R Schober, Max-max relay selection for relays with buffers. IEEE Trans. Wirel. Commun. 11(3), 1124-1135 (2012)

28. I Krikidis I, T Charalambous, JS Thompson, Buffer-aided relay selection for cooperative diversity systems without delay constraints. IEEE Trans. Wirel. Commun. 11(5), 1957-1967 (2012)

29. Z Tian, G Chen, Y Gong, Z Chen, J Chambers, Buffer-aided max-link relay selection in amplify-and-forward cooperative networks. IEEE Trans. Veh. Technol. 64(2), 553-565 (2014)

30. N Nomikos, T Charalambous, I Krikidis, D Vouyioukas, M Johansson, Hybrid cooperation through full-duplex opportunistic relaying and max-link relay selection with transmit power adaptation, in Proc. of Communications (ICC) 2014 IEEE International Conference on, 2014, pp. 5706-5711

31. C Gaojie, T Zhao, G Yu, J Chambers, Decode-and-forward buffer-aided relay selection in cognitive relay networks. IEEE Trans. Veh. Technol. 63(9), 4723-4728 (2014)

32. B Vo Nguyen Quoc, TQ Duong, DB da Costa, GC Alexandropoulos, A Nallanathan, Cognitive amplify-and-forward relaying with best relay selection in non-identical Rayleigh fading. IEEE Commun. Lett. 17(3), 475-478 (2013)

33. HA David, HN Nagaraja, Order statistics, 3rd edn. (John Wiley, New York, 2003)

34. IS Gradshteyn, IM Ryzhik, Table of integrals, series, and products, 7th edn. (Acdemic, San Diego, 2007)

35. TQ Duong, VNQ Bao, HJ Zepernick, On the performance of selection decode-and-forward relay networks over Nakagami-m fading channels. IEEE Commun. Lett. 13(3), 172-174 (2009)

36. GC Alexandropoulos, A Papadogiannis, K Berberidis, Performance analysis of cooperative networks with relay selection over Nakagami- $m$ fading channels. IEEE Signal Process Lett. 17(5), 441-444 (2010)

\section{Submit your manuscript to a SpringerOpen ${ }^{\circ}$ journal and benefit from:}

- Convenient online submission

- Rigorous peer review

- Immediate publication on acceptance

- Open access: articles freely available online

- High visibility within the field

- Retaining the copyright to your article

Submit your next manuscript at $>$ springeropen.com 